\title{
Acknowledgement to Reviewers of the International Journal of Molecular Sciences in 2017
}

\author{
International Journal of Molecular Sciences Editorial Office
}

MDPI AG, St. Alban-Anlage 66, 4052 Basel, Switzerland

Published: 19 January 2018

Peer review is an essential part in the publication process, ensuring that the International Journal of Molecular Sciences maintains high quality standards for its published papers. In 2017, a total of 2778 papers were published in the journal. Thanks to the cooperation of our reviewers, the median time to first decision was 20 days and the median time to publication was 43 days. The editors would like to express their sincere gratitude to the following reviewers for their time and dedication in 2017:

Abbott, Karen L.

Abdalla, Maher Y.

Abd-El-Aziz, Alaa

Abenavoli, Ludovico

Abend, Michael

Abizaid, Alfonso

Abou Neel, Ensanya A.

Abraham, Dietmar

Abuelo, Ángel

Aburima, Ahmed

Abu-Romman, Saeid

Accornero, Federica

Achinger-Kawecka, Joanna

Achour, Brahim

Acuña-castroviejo, Dario

Adachi, Hiroaki

Adam, Virgile

Adam, Vojtech

Adams, James

Adams, Michael A.

Adan, Roger

Addis, Maria Filippa

Adhikari, Neeta

Adunyah, Samuel E.

Aeby, Eric

Afosah, Daniel

Afrin, Sadia

Agarwal, Gaurav
Lopes, Susana

Lopez De Diego, Marta

López, Alicia

Lopez, José Manuel Blanco

López-Andrés, Natalia

Lopez-Camarillo, Cesar

Lopez-Escamez, Jose A.

López-Moya, Juan José

Lophatananon, Artitaya

Lora, Jairo

Lord, Janet

Lorenzini, Antonello

Lorenzo, Óscar

Lorey, Fred

Lorico, Aurelio

Lorson, Christian

Lortat-Jacob, Hugues

Los, Marek

Lossius, Andreas

Lötsch, Jörn

Lou, Song

Lou, Yonggen

Loutradis, Dimitrios

Louveau, Isabelle

Love, John

Lowe, Martin

Lowe, Rohan

Loxham, Matthew 
Agarwal, Shivangi

Ageitos, Jose

Aggarwal, Abhishek

Aggarwal, Bharat B.

Agostoni, Carlo

Agudelo-Romero, Patricia

Aguilo, Francesca

Ah Kim, Hyun

Ahmad, Zeeshan

Ahmadi-Afzadi, Masoud

Ahmed, Hafiz

Ahmed, Marya

Ahn, Ji-Young

Ahnert, Peter

Ahrens-Nicklas, Rebecca C.

Aimanianda, Vishu Kumar

Airas, Laura

Aires, Alfredo

Airoldi, Cristina

Aithal, Guru

Aitor, Nogalez-Gonzalez

Akamatsu, Miki

Akhtari, Massoud

Akiba, Etsuko

Akimov, Sergey A.

Akita, Sadanori

Al Massadi, Omar

Ala, Ugo

Al-Aubaidy, $\mathrm{H}$.

Alavez, Silvestre

Albano, Emanuele

Albi, Elisabetta

Albrecht, Eric A.

Albrecht, Randy

Alcaide, Pilar

Aldamiz-Echevarria, Luis

Al-Dujaili, Emad

Alemany, Silvia

Alessandro, Riccardo

Alexander, Denis

Alexandre, Mezentsev

Alexeyev, Mikhail

Alexeyev, Mikhail F.
Lozano, Jose Manuel

$\mathrm{Lu}$, Jun

Lu, Linchao

Lu, Shuo

Lu, Wan-Jung

Lu, Yang

Lu, Zhanping

Luca, Maria

Lucacchini, Antonio

Lucarini, Guendalina

Luce-Fedrow, Alison

Lucena, María Isabel

Lucena, Rafael

Luchetti, Michele

Luciano, Alberto Maria

Luckenbach, Till

Luco, Reini F.

Luconi, Michaela

Lüder, Carsten G. K.

Ludueña, Richard

Ludwiczuk, Agnieszka

Luft, Thomas

Luís, Ângelo

Lukas, Jan

Lukashevich, Igor S.

Lukaszewski, Adam

Lundstrom, Kenneth

Luo, Huacheng

Luo, Lyna

Luongo, Livio

Lupien, Andréanne

Luque, Francisco

Łuszczek-Trojnar, Ewa

Lutfy, Kabirullah

Luthra, Amit

Luu, Doan-trung

Ly, Hinh

Lymperopoulos, Anastasios

Lynce, Filipa

Lyng, Maria B

Lyons, Lisa

Lyons, Paul E.

Lystad, Alf Håkon 
Alexiou, Christoph

Alfano, Daniela

Alfano, Massimo

Al-Hendy, Ayman

Alique, Matilde

Alivernini, Stefano

Allardyce, Claire

Allegra, Sarah

Allen, Christopher

Allen, Margaret L.

Allione, Alessandra

Allnutt, Theo

Almasan, Alex

Al-Mashhadi, Rozh H.

Almeida, Pedro

Almouzni, Genevieve

Alonso, Santos

Alonso-Magdalena, Paloma

Alshbool, Fatima Z.

Altaner, Cestmir

Alteri, Christopher J.

Altieri, Barbara

Altomare, Claudia

Altomonte, Jennifer

Alvarez, Catalina

Alves, Celso

Alves, Sandra

Alwahsh, Salamah

Alwood, Joshua S.

Amaral, Alexandra

Amarowicz, Ryszard

Amasheh, Salah

Amati, Francesca

Ambati, Ranga Rao

Ambigapathy, Ganesh

Ameri, P.

Amicarelli, Fernanda

Amini-Nik, Saeid

Amirkhani, Masoume

Amit, Tiwari

Amital, Howard

Amorim, Maria João

Amorós, Asunción
M. Peffley, Dennis

M.Fuller, Patrick

M.L. Hutnik, Cindy

Ma, Guojia

Ma, Qing

Ma, Wen-Juan

Ma, Xiaochao

Ma, Xingzhe

Ma, Yonggang

$\mathrm{Ma}, \mathrm{Yu}$

Ma, Zexu

Maak, Steffen

Macabeo, Allan P.

Macchi, Beatrice

MacDonald, Clinton C.

Macdonald, Stephen P. J.

Macedo, Rodrigo

Machado, Mariana V.

Machireddy, Narsa

Macia, Angela

Macias, Maria J.

Maciejewska, Dominika

Mack, Andreas F.

Macvittie, Thomas J.

Madabhushi, Ram

Madan, Juliette

Madeleine, Margaret M.

Madhukar, Burra

Maeda, Hiroshi

Maekawa, Fumihiko

Maekawa, Shinya

Maestro, Miguel A.

Magnaghi, Valerio

Magnaldo, Thierry

Magni, Fulvio

Magnusson, Karl-Eric

Mahajan, Sahil

Mahajan, Vinit B.

Mahajna, Jamal

Mahimainathan, Lenin

Mahmood, Javed

Mahoney, Sara E.

Mahony, Timothy John 
Ampatzis, Konstantinos

Ampuero, Javier

Amreddy, Narsireddy

An, Sai

Ananthakrishnan, Soundaram Jeevarathinam

Anastasi, Emanuela

Anastasiadou, Maria

Anczukow-Camarda, Olga

Andersen, Thomas Levin

Anderson, Travis M.

Andersson, Emma

Andjelkovic, Anuska V.

Ando, Hitoshi

Andolfo, Annapaola

André, Vânia

Andreassi, Maria Grazia

Andreasson, Erik

Andreoni, Francesca

Andreozzi, Giuseppe Maria

Andres, Allen M.

Andrés, Maria Fe

Andrews, Zane

Andrey, Guillaume

Andrieux, Annie

Andrukhov, Oleh

Anes, Elsa

Angelo, Alberti

Angelova, Angelina

Angelovich, Tom

Anglister, Jacob

Aniello, Francesco

Anifandis, George

Anjos, Ofélia

Ankiewicz, Adrian

Annadurai, Anandhan

Anower, Rokebul

Ansquer, Jean Claude

Antal, Diana Simona

Antaris, Alexander Lee

Anthony, Karen

Antognelli, Cinzia

Antoine, Marie-Hélène

Anton, Steve
Mahsoub, Hassan

Maier, Patrick

Maillard, Virginie

Maioli, Margherita

Maione, Francesco

Mai-Prochnow, Anne

Maiti, Aparna

Majumdar, Indrajit

Majumdar, Sudipta

Majumder, Mousumi

Makarevitch, Irina

Makena, Monish

Makishima, Makoto

Malemud, Charles J.

Malentacchi, Francesca

Malhotra, Vivek

Malkoc, Veysi

Malli, Roland

Mallipeddi, Prema Latha

Mallo, Federico

Malloci, Giuliano

Malmström, Lars

Maloyan, Alina

Malpeli, Giorgio

Mamedov, Tarlan

Mammoto, Tadanori

Man, Dula

Mancheño, José

Mancias, Joseph D.

Mandapati, R. R.

Mandava, Nanda

Mandolesi, Georgia

Mandrioli, Mauro

Mangiatordi, Giuseppe Felice

Mann, Francis

Mann, Karen P.

Mannell, Hanna

Manni, Andrea

Manninger, Martin

Manno, Carlo

Manocha, Gunjan

Manor, Danny

Mansky, Kim C. 
Antonelou, Marianna H.

Antoniadi, Ioanna

Antoniak, Silvio

Anzenbacher, Pavel

Aon, Miguel A.

Aoyama, Koji

Aparicio, Frederic

Apone, Fabio

Applegate, Lee Ann

Apte, Udayan

Ardley, Julie

Arduini, Alessandro

Arena, Francesca

Arenas, Jesús

Arens, Paul

Arent, Camila

Arese, Marzia

Argia, Katsuhiko

Arianna, Marucco

Ariga, Hiroyoshi

Ariyasu, Daisuke

Ariza, Maria Eugenia

Arnbjörnsson, Einar

Arndt, Patrick G.

Arnould, Thierry

Aronowitz, Joel A.

Aronson, Melyssa

Arrabal, Pilar

Arteaga, Olatz

Artioli, Grazia

Artlip, Timothy

Arulselvan, Palasamy

Arumugam, Somasundaram

Asa, Sylvia

Asahara, Hiroshi

Asahi, Michio

Asamizu, Shumpei

Asano, Ryutaro

Asano, Tomoichiro

Asatryan, Rubik

Asensi, Victor

Ashiuchi, Makoto

Askarian-Amiri, Marjan E.
Mantzioris, Evangeline

Manzardo, A.M.

Manzella, Livia

Mao, Meng

Marasco, Daniela

Marc, Daniel

Marcal, Helder

Marchant, David

Marchese, Adriano

Marchetti, Marta

Marchini, Sergio

Marci, Roberto

Marcinkowska, Ewa

Marco, Eva María

Marcovici, Geno

Mardente, Stefania

Maréchal, Alexandre

Maréchal-Drouard, Laurence

Márialigeti, Károly

Marikawa, Yusuke

Marin, Jose J. G.

Marincs, Ferenc

Mariniello, Loredana

Marino, Angela

Marino, Joseph

Maritschnegg, Elisabeth

Markiv, Anatoliy

Markkanen, Enni

Marklund, Ulrika

Markov, Marko S.

Marlow, Florence L.

Marnetto, Fabiana

Marrs, James A.

Marsich, Eleonora

Marsolais, Frédéric

Martí, Sergio

Martin, Abraham

Martin, Cathie

Martin, Finian

Martin, Francisco

Martin, Jessica

Martin, Luc J.

Martin, Thomas John 
Aslam, Muhammad

Aslanidis, Charalampos

Assanelli, Deodato

Assenat, Eric

Astolfi, Stefania

Asuero, Agustín G.

Ates-Alagoz, Zeynep

Athyros, Vasilios

Attanasio, Chiara

Attar, Hooyar

Atteritano, Marco

Attila, Adam

Audette, Gerald F.

Audrey, Varin

Augé, Nathalie

Auguet, Teresa

Augusto, Orlandi

Austin, Rachel

Auvynet, Constance

Avato, Pinarosa

Avci-Adali, Meltem

Averbeck, Dietrich

Aviles, Francesc Xavier

Avni, Adi

Avrova, Natalia F.

Ay, Ferhat

Aydemir, Tolunay

Ayers, Duncan

Ayuso, Maria Irene

Azarnia Tehran, Domenico

Azevedo, Herlander

Azhar, Mohamad

Azimzadeh, Omid

Azuma, Kagaku

Azuma, Kazuo

Baba, Hideo

Baba, Yuh

Babu, Anish

Bach, Andreas

Bache, Søren

Back, K.

Backer, Marina V.

Backman, Ludvig
Martina, Korfei

Martín-Acebes, Miguel A.

Martín-Antonio, Beatriz

Martín-Aragón, Sagrario

Martineau, Yvan

Martinelli, Paola

Martínez De Paz, Pedro José

Martínez, Antonio

Martinez, Manuel

Martinez, Vicente

Martínez-Campa, Carlos

Martínez-Esteso, María José

Martínez-Gómez, Pedro

Martinez-Lage, Andres

Martin-Fontecha, Alfonso

Martín-Hernández, David

Martini, Claudia

Martini, Maurizio

Martínková, Ludmila

Martino, Sabata

Martinoli, Maria-Grazia

Martins, Albino

Martins, Ian James

Martins, Natália

Martorana, Alessandro

Maruf, Abdullah Al

Marullo, Stefano

Maruyama, Kei

Marverti, Gaetano

Marvin, Shauna

Marycz, Krzysztof

Marzban, Hassan

Marzocco, Stefania

Marzullo, Paolo

Masaki, Tsutomu

Maserti, Bianca Elena

Maserti, Biancaelena

Mashima, Ryuichi

Masiello, Pellegrino

Masieri, Federica

Masli, Sharmila

Masłyk, Maciej

Masquida, B. 


\begin{tabular}{|c|c|}
\hline Baczek-Kwinta, Renata & Massot, Malen \\
\hline Bado, Igor & Masters, Colin L. \\
\hline Bae, Hae-Rahn & Mastrangelo, Anna M. \\
\hline Baek, Kwang-Hyun & Mastrocola, Raffaella \\
\hline Baenas, Nieves & Mastropasqua, Leonardo \\
\hline Baer, Patrick C. & Masuda, Kiyoshi \\
\hline Baeumer, Wolfgang & Masuda, Shinji \\
\hline Bagchi, Rammyani & Masui, Kenta \\
\hline Bahar, Entaz & Masureel, Matthieu \\
\hline Bai, Hua & Mateos-Timoneda, M. A. \\
\hline Bai, Li-Yuan & Mateu, Enric \\
\hline Baier, Margarete & Mathesius, Ulrike \\
\hline Bairaktari, Eleni T. & Matheu, Ander \\
\hline Bajpai, Richa & Matic, Maja \\
\hline Bakay, Marina & Matos, Augusto J. F. \\
\hline Bakovic, Marica & Matos, Manuela \\
\hline Baksh, Shairaz & Matsubara, Keiichi \\
\hline Bakunina, Irina & MATSUGAKI, Aira \\
\hline Bal, Wojciech & Matsui, Masaki \\
\hline Balato, Anna & Matsumoto, Ken-ichiro \\
\hline Baldini, Enke & Matsumoto, Kunio \\
\hline Baldwin, Cynthia & Matsumoto, Yasuhiko \\
\hline Balestrini, Raffaella Maria & Matsuo, Muneaki \\
\hline Ball, Jonathan & Matsushita, Takehiko \\
\hline Ballerini, Clara & Matsuura, Bunzo \\
\hline Ballizany, Wouter & Matta, Csaba \\
\hline Bally, Julia & Matta, Jaime \\
\hline Bally, Marta & Mattei, Benedetta \\
\hline Bamdad, Cynthia & Mattei, Fabrizio \\
\hline Bamford, Connor & Matteoli, Gianluca \\
\hline Bandini, Erika & Mattner, Jochen \\
\hline Banerjee, Hirendra Nath & Matwijczuk, Arkadiusz \\
\hline Banerjee, Kalpita & Maura, Damien \\
\hline Banerjee, Partha & Mauriello, Francesco \\
\hline Banfalvi, Zsofia & Maury, Eleonore \\
\hline Bang, Marie-Louise & Mautner, Andreas \\
\hline Baniahmad, Aria & Mavridis, Konstantinos \\
\hline Banilas, Georgios & Mavrogonatou, Eleni \\
\hline Bannard-Smith, Jonathan & Mawhinney, Thomas \\
\hline Banning, Antje & Maxel, Trine \\
\hline Bansal, Ruchi & Maximiano, Marisa Da Silva \\
\hline Banti, Christina N. & Maxwell, Anthony \\
\hline Bao, Yongping & Maxwell, Jessie R. \\
\hline
\end{tabular}




\begin{tabular}{|c|c|}
\hline Baptiste-Roberts, Kesha & May, Felicity \\
\hline Barabas, Peter & Mayer, Gregory D. \\
\hline Barabutis, Nektarios & Mayhan, William G. \\
\hline Baragetti, Andrea & Mayne, Christopher \\
\hline Barata, João & Mazur-Bialy, Agnieszka Irena \\
\hline Barbagallo, Davide & McArdle, Stephanie \\
\hline Barbe, Anna Greta & McCallum, Jason L. \\
\hline Barbe, Mary F. & McCormick, Craig \\
\hline Barber, Melissa & McCoy, Sarah J. Breese \\
\hline Barbieri, Antonio & McDaid, Hayley \\
\hline Barbu, Sorin T. & McDaneld, Tara G. \\
\hline Barcelos, Gustavo & McDermott, Lindsay \\
\hline Bárcena, José Antonio & McDermott-Roe, Chris \\
\hline Barchetta, Ilaria & McDonagh, Brian \\
\hline Bärebring, Linnea & Mcdonald, Courtney \\
\hline Barilli, Amelia & McDonald, Karen \\
\hline Barisani, Donatella & McFeeters, Robert L. \\
\hline Barrajón-Catalán, Enrique & McGowan, Eileen \\
\hline Barrea, Luigi & McGrail, Maura \\
\hline Barros, Pedro & Mcintosh, Robert \\
\hline Barry, Carol & McIntyre, Jenifer K. \\
\hline Barta, Csengele & McKay, Brian \\
\hline Bartelt, Alexander & McKeague, Maureen \\
\hline Bartholomeusz, Geoffrey & McKenna, Malachi J. \\
\hline Bartley, James & McKim, Kim S. \\
\hline Bartocci, Pietro & McLean, Robert J. C. \\
\hline Bartolazzi, Armando & McLemore, Gabrielle Lynn \\
\hline Bartolini, Manuela & Mcloughlin, P. \\
\hline Bartosz, Grzegorz & McMahon, Francis \\
\hline Bartoszewski, Rafal & McMahon, Hilary E.M. \\
\hline Barzilay, Joshua I. & McMenamin, Mark \\
\hline Bar-Zvi, Dudy & McMillin, Matthew \\
\hline Basak, Debasish & Mcmorrow, Tara \\
\hline Basar, Murat & McMurray, Fiona \\
\hline Basavarajappa, Balapal S. & Meacci, Elisabetta \\
\hline Bascom, Gavin & Meade, Kieran \\
\hline Basiak, Ewelina & Meadows, Shannon \\
\hline Basini, Giuseppina & Mediati, Rocco Domenico \\
\hline Basolo, Fulvio & Medina Piles, Vicente \\
\hline Basso, Kari & Mehta, Akul Y. \\
\hline Bastarrachea, Luis J. & Mehta, Pooja \\
\hline Basu, Abhijit & Mehta, Shwetal \\
\hline Basu, Alakananda & Mehta, Stuti \\
\hline
\end{tabular}




\begin{tabular}{|c|c|}
\hline Basu, Sujit & Mei, Ya-Fang \\
\hline Batorova, Angelika & Meierhofer, David \\
\hline Batsché, Eric & Meijer, H. J. G. \\
\hline Battino, Maurizio & Meijles, Daniel N. \\
\hline Battistelli, Cecilia & Meinke, Peter \\
\hline Battistelli, Michela & Melchor, Juan \\
\hline Battisti, James & Meldolesi, Jacopo \\
\hline Baucher, Marie & Meli, Albano \\
\hline Baud, Olivier & Melis, Anastasios \\
\hline Bauer, Georg & Melkani, Girish \\
\hline Bauer, Johann & Mellor, Andrew L. \\
\hline Baulch, Janet E. & Melnik, Bodo \\
\hline Bawadekar, Mandar & Melo, Sonia \\
\hline Bayat, Vafa & Meloni, Gabriele \\
\hline Bayin, N. Sumru & Melville, Stephen \\
\hline Bazafkan, Hoda & Menand, Benoît \\
\hline Bazhanova, Elena D. & Menassa, Rima \\
\hline Bazihizina, Nadia & Mendonca, Antonio \\
\hline Bažok, Renata & Mendonça, Nuno \\
\hline Beatriz, Abós Gracia & Menet, Marie Claude \\
\hline Beatson, Richard E. & Meng, Dong \\
\hline Beberok, Artur & Meng, Weihua \\
\hline Beck, Andreas & Menkhorst, Ellen \\
\hline Beckenbach, Andrew & Menon, Vipin \\
\hline Becker, Christoph & Menu, Eline \\
\hline Becker, Kathrin & Mercati, Francesco \\
\hline Becker, Therese M. & Merry, Troy \\
\hline Becuwe, Philippe & Meruvu, Sunitha \\
\hline Bedia, Carmen & Meschwitz, Susan \\
\hline Bedogni, Francesco & Messa, Piergiorgio \\
\hline Beer, Ross & Messaritakis, Ippokratis \\
\hline Behera, Smrutisanjita & Messini, Christina I. \\
\hline Beilby, Mary & Messinis, Ioannis \\
\hline Beisswenger, Christoph & Messner, Barbara \\
\hline Beitz, Eric & Metharom, Pat \\
\hline Bejarano, Ignacio & Mettananda, Sachith \\
\hline Bekinschtein, Pedro & Metzinger, Laurent \\
\hline Bekri, Soumeya & Meulia, Tea \\
\hline Belden, William J. & Meurens, François \\
\hline Belgacem, Yesser H. & Mexas, Angela Marie \\
\hline Belide, Srinivas & Meyer, Gregory \\
\hline Bell, Andrew & Mézes, Miklós \\
\hline Bellés, José María & Mező, Gábor \\
\hline
\end{tabular}




\begin{tabular}{|c|c|}
\hline Bellosta, Paola & Miao, Ji \\
\hline Belluzzi, Andrea & Miao, Yinglong \\
\hline Belov, George & Miccadei, Stefania \\
\hline Bełtowski, Jerzy & Michael, Groszmann \\
\hline Belyaeva, Natalia & Michaeloudes, Charalambos \\
\hline Ben Mahmoud, Lobna & Michael-Titus, Adina Teodora \\
\hline Benaiges, Maria D. & Michalek, Katarzyna \\
\hline Ben-Dov, Iddo Z. & Michalska, Anna \\
\hline Benedetti, Michele & Micheau, Olivier \\
\hline Benigni, Ariela & Micheletti, Gabriele \\
\hline Benjamin, Don & Michelhaugh, Sharon Kay \\
\hline Benner, Chris & Michetti, Fabrizio \\
\hline Benno, Weigmann & Michiels, Carine \\
\hline Benoist, Hervé & Michigami, Toshimi \\
\hline Benowitz, Larry I. & Mico, Juan Antonio \\
\hline Bentel, Jacqueline M. & Midgley, Adam \\
\hline Benzeroual, Kenza E. & Midgley, Adam C. \\
\hline Berbari, Nicolas & Mieda, Michihiro \\
\hline Berdis, Anthony J. & Miernyk, Jan A \\
\hline Berecki, Geza & Mietlicki-Baase, Elizabeth G. \\
\hline Berenbrink, Michael & Miguel, Leiva-Brondo \\
\hline Berent-Maoz, Beata & Míguez, Jesús M. \\
\hline Bergen, Werner & Mihailiasa, Manuela \\
\hline Berger, Ralf G. & Mi-ichi, Fumika \\
\hline Bergeron, E. & Mikel, Zaratiegui \\
\hline Bergheim, Ina & Mikhailova, Alexandra \\
\hline Bergquist, Robert & Miki, Koji \\
\hline Bermudez, Marcel & Miki, Yasuhiro \\
\hline Bernardi, Francesco & Miklossy, Gabriella \\
\hline Bernasconi, Pia & Mikula, Mario \\
\hline Bernitsas, Evanthia & Milanesio, Marco \\
\hline Bernstein, Hans-Gert & Milano, Francesco \\
\hline Berruyer, Romain & Milej, Daniel \\
\hline Berski, Wiktor & Milhinhos, Ana \\
\hline Berthelot, Karine & Milic, Natasa \\
\hline Berthelot, Laureline & Milione, Massimo \\
\hline Berthod, François & Millar, J. Cameron \\
\hline Bertinaria, Massimo & Miller, Aaron W. \\
\hline Bertok, Tomas & Miller, John \\
\hline Bertoli, Cosetta & Miller, Judith S. \\
\hline Bertoli, Gloria & Millet, Oscar \\
\hline Bertolini, Marta & Mills, Stuart \\
\hline Bertoni, Giuseppe & Milner, Joel \\
\hline
\end{tabular}




\begin{tabular}{|c|c|}
\hline Besnard, Guillaume & Mimaki, Yoshihiro \\
\hline Bevan, David & Min, Baehyun \\
\hline Beverly, Levi J. & Min, Jung-joon \\
\hline Beversdorf, David & Minami, Yoichi \\
\hline Beyer, Andreas M. & Minchin, James \\
\hline Bhadauria, Vijai & Mine, Yuichi \\
\hline Bhattacharyya, Gargi & Minervini, Giovanni \\
\hline Bhaumik, Anusarka & Ming-Wei, Lin \\
\hline Bhuiyan, Md. Shenuarin & Mino, Masanobu \\
\hline Bhutani, Nidhi & Minocha, Subhash C. \\
\hline Bhutta, Mahmood & Minor, Philip \\
\hline $\mathrm{Bi}, \mathrm{Xin}$ & Minutolo, Filippo \\
\hline Biagi, Marco & Miotto, Benoit \\
\hline Biagini, Giuseppe & Miousse, Isabelle \\
\hline Bian, Li & Miquel, M. \\
\hline Biasi, Fiorella & Miragoli, Michele \\
\hline Bichet, Daniel G. & Mirali, Mahla \\
\hline Bichko, Vadim & Miranda, Cristobal \\
\hline Bicker, Gerd & Miranda-Castro, Rebeca \\
\hline Bickerton, Erica & Mireau, Hakim \\
\hline Bid, Hemant & Mirey, Gladys \\
\hline Biddle, Adrian & Miri, Amir K. \\
\hline Bideshi, Dennis & Miron, Richard J. \\
\hline Bidwai, Ashok & Miroshnichenko, Andrey \\
\hline Biedermann, Thomas & Mirzayans, Razmik \\
\hline Bielak-Zmijewska, Anna & Mishin, Alexander S. \\
\hline Bienkiewicz, Ewa Anna & Mishra, Jayshree \\
\hline Biernasiuk, Anna & Mishra, Nigam \\
\hline Biersack, Bernhard & Miteva, Mariya \\
\hline Bijak, Michał & Mithöfer, Axel \\
\hline Bijlard, Eveline & Mitoma, Hiroki \\
\hline Bijlsma, Maarten F. & Mitra, Abhisek \\
\hline Bikiaris, Dimitrios & Mitrofanis, John \\
\hline Bilbao, Jose Ramon & Mitroulis, Ioannis \\
\hline Bilger, Andrea & Mitsuya, Shiro \\
\hline Bingol, Kerem & Miura, Kazuki \\
\hline Binstadt, Bryce & Miura, Sayaka \\
\hline Biondani, Giulia & Miyaji, Hirofumi \\
\hline Birch, John & Miyake, Makito \\
\hline Bird, Amanda & Miyamoto, Koji \\
\hline Bird, Ranjana & Miyamoto, Shingo \\
\hline Birkemeyer, Claudia & Miyanoiri, Yohei \\
\hline Birrell, Mark & Miyata, Atsuro \\
\hline
\end{tabular}




\begin{tabular}{|c|c|}
\hline Bisaga, Maciej & Miyata, Yoshihiko \\
\hline Bisaglia, Marco & Miyoshi, Hiroaki \\
\hline Bischof, Joachim & Miyoshi, Norio \\
\hline Bishehsari, Faraz & Mizobata, Tomohiro \\
\hline Bishop, Douglas & Mizuno, Cassia S. \\
\hline Bisignano, Giuseppe & Mizuno, Masashi \\
\hline Bišová, Kateřina & Mlejnek, Petr \\
\hline Bisson, Jonathan & Mlera, Luwanika \\
\hline Biswas, Santanu & Mo, Jung-Soon \\
\hline Bitler, Benjamin & Moal, Iain \\
\hline Bittner, Stefan & Moar, William J. \\
\hline Bizzarri, Mariano & Mobed-Miremadi, Maryam \\
\hline Björnsson, Einar Stefán & Mocan, Andrei \\
\hline Black, Samuel J. & Mocellin, Simone \\
\hline Blais, Anne & Modahl, Cassandra \\
\hline Blanco-Aparicio, Carmen & Modhiran, Naphak \\
\hline Blanco-Ulate, Barbara & Modos, Dezsö \\
\hline Blando, Federica & Moeller, Hanne B. \\
\hline Blank, Volker & Moertl, Simone \\
\hline Blankesteijn, W. Matthijs & Mogi, Masaki \\
\hline Blasiak, Janusz & Mohamed, Islam \\
\hline Blatt, Michael & Mohamedali, Khalid \\
\hline Blomme, Jonas & Mohammad, Haroon Taj \\
\hline Blum, Robert & Mohammed-Geba, Khaled \\
\hline Blumenthal, Edward & Mohanta, Tapan \\
\hline Blumer-Schuettea, Sara E. & Möhlhenrich, S. C. \\
\hline Boareto Do Amaral, Marcelo & Mohr, Stefan \\
\hline Bobek, Vladimir & Mok, Daniel Kam-Wah \\
\hline Bocchio-Chiavetto, Luisella & Moldawer, Lyle L. \\
\hline Boccuto, Luigi & Molfino, Alessio \\
\hline Bochman, Matthew L. & Mollapour, Mehdi \\
\hline Boczek, Tomasz & Mollen, Kevin P. \\
\hline Bode, Robert F. & Møller, Ian Max \\
\hline Bodnar, Lubomir & Molnar, Ferdinand \\
\hline Boege, Fritz & Molugu, Sudheer Kumar \\
\hline Boekema, Bouke & Molyneux, Karen \\
\hline Boerma, Marjan & Moncayo, Roy \\
\hline Bogado Pascottini, Osvaldo & Monchau, Francine \\
\hline Bogdanowicz, Krzysztof & Mongiat, Maurizio \\
\hline Bogdański, Paweł & Monk, Jennifer \\
\hline Boggaram, Vijay & Monroy, Fernando \\
\hline Bogliolo, Massimo & Montani, Jean-Pierre \\
\hline Bogni, Alessia & Monteiro, Susana \\
\hline
\end{tabular}




\begin{tabular}{|c|c|}
\hline Bøgwald, Jarl & Monteith, G. R. \\
\hline Bohlson, Suzanne S. & Montenegro, Lucia \\
\hline Bohutskyi, Pavlo & Montesarchio, Daniela \\
\hline Boisvert, François-Michel & Monteys, Alex Mas \\
\hline Bojková, Bianka & Montoya, Guillermo \\
\hline Bolger, Anthony & Moon, Byeong Cheol \\
\hline Bolibok-Brągoszewska, H. & Moon, Ilsoo \\
\hline Bolino, Alessandra & Moore, Axel C. \\
\hline Bollag, Wendy B. & Moore, Thomas F \\
\hline Bolle, Cordelia & Moos, Philip \\
\hline Bölter, Bettina & Morales, Julio \\
\hline Bombieri, Cristina & Morales, Paula \\
\hline Bonen, Linda & Morales-Cruz, Abraham \\
\hline Bongers, Kale & Morales-González, J. A. \\
\hline Boogaard, Peter & Morales-Tirado, Vanessa \\
\hline Boomer, Jonathan S. & Morandi, Luca \\
\hline Bopegamage, Shubhada & Morbidelli, Lucia \\
\hline Borges, João P.M.R. & Moreira, Irina \\
\hline Borges, Karin & Moreira-Teixeira, Lúcia \\
\hline Borghaei, Ruth C. & Moreno, Carlos S. \\
\hline Borghini, Andrea & Moreno, Margarita \\
\hline Borkow, Gadi & Moreno, Maria \\
\hline Borkowski, Leszek & Morgat, Clément \\
\hline Borodinsky, Laura N. & Mori, Kazuhiro \\
\hline Borovecki, Ana & Moriarty, Thomas Fintan \\
\hline Borrego, Francisco & Moriguchi, Takaya \\
\hline Borroni, Riccardo & Morikawa, Satoru \\
\hline Borst, Piet & Morikawa, Toshio \\
\hline Borucki, Monica & Morimoto, Tatsuya \\
\hline Boryczka, Stanisław & Morino, Katsutaro \\
\hline Boscaiu, Monica & Morioka, Tomoaki \\
\hline Boschetti, Federica & Morita, Hiroyuki \\
\hline Bose, Debojit & Morita, Manabu \\
\hline Bose, Sayantan & Morley, Alexander A. \\
\hline Bosman, Giel J. C. G. M. & Morley, Steven Douglas \\
\hline Bosnjak, Berislav & Moro, Loredana \\
\hline Bosse, Jens B. & Moro, Stefano \\
\hline Bossi, Fleur & Morotti, Alessandro \\
\hline Botelho, Monica C. & Morreale, Marco \\
\hline Botha, Frederik & Morris, Andrew J. \\
\hline Boto, Luis & Morris, Claudia R. \\
\hline Bottari, Fabio & Morris, James L. \\
\hline Bottaro, Donald P. & Morrison, Douglas \\
\hline
\end{tabular}




\begin{tabular}{|c|c|}
\hline Bottier, Céline & Morrison, Melanie \\
\hline Bouattour, Mohamed & Morris-Rosendahl, Deborah \\
\hline Bouckaert, Julie & Morrissey, Jeremiah \\
\hline Bourdon, Emmanuel & Morroni, Fabiana \\
\hline Bouropoulos, Nikolaos & Morrow, Jennifer \\
\hline Bousbaa, Hassan & Mortiboys, Heather \\
\hline Boutin, Jean & Mortier, Jérémie \\
\hline Boutin, Jean Albert & Morton, David \\
\hline Bouttier, Manuella & Mortusewicz, Oliver \\
\hline Bouvier, Benjamin & Moschetta, Michele \\
\hline Bowen, James & Moschovi, Maria \\
\hline Bowen, Richard & Moser, Lindsey A. \\
\hline Bowerman, Melissa & Mossialos, Dimitris \\
\hline Bowling, Heather & Motta, Antonella \\
\hline Bowsher, Alan W. & Mottes, Monica \\
\hline Boyd, Chris & Mourad, Raphael \\
\hline Boyer-Guittaut, Michaël & Mourtas, Spyros \\
\hline Boysen, Preben & Mousa, Shaaban A. \\
\hline Bozic, Josko & Moustaid-Moussa, Naima \\
\hline Bozzaro, Salvatore & Movahedi, Ali \\
\hline Bozzi, Adriana & Moxon, Joseph \\
\hline Bozzi, Fabio Luigi & Muckle, Catherine Anne \\
\hline Brábek, Jan & Mueller, Geoffrey \\
\hline Bracci, Massimo & Mueller, Wolf C. \\
\hline Bradbury, Allison & Muenzer, Joseph \\
\hline Bramanti, Vincenzo & Mufson, Elliott J. \\
\hline Branchini, Alessio & Muggia, Franco \\
\hline Brandão, Rita Dias & Muinelo-Romay, Laura \\
\hline Brandhorst, Bruce & Mukaida, Naofumi \\
\hline Bratlie, Kaitlin M. & Mukherjee, Somnath \\
\hline Bravo López, Susana B. & Mulas, Maurizio \\
\hline Breitenbach Barroso Coelho, Luana Cassandra & Mulatero, Paolo \\
\hline Breitenfeld Granadeiro, Luiza & Mulinacci, Nadia \\
\hline Brender, Jeffrey & Müller, Markus \\
\hline Brennan, Stephen & Muller, Patricia A. J. \\
\hline Brenner, Thorsten & Müller, Timo \\
\hline Brestic, Marian & Mulo, Paula \\
\hline Breves, Gerhard & Munang'andu, H. M. \\
\hline Brewer, Megan & Munday, Michael \\
\hline Bricknell, Ian & Muñoz-Barroso, Isabel \\
\hline Bridges, Christy & Munoz-Fontela, Cesar \\
\hline Bridgewater, Darren & Munro, Carol \\
\hline Brier, Michael E. & Mura, Umberto \\
\hline
\end{tabular}




\begin{tabular}{|c|c|}
\hline Brierley, Ian & Murai, Junko \\
\hline Brinchmann, Monica F. & Murakami, Seishi \\
\hline Brini, Marisa & Murakami, Yasufumi \\
\hline Brochot, Amandine & Murakami, Yoshiki \\
\hline Broderick, Tom L. & Murase, Sachiko \\
\hline Bröer, Stefan & Murata, Takayuki \\
\hline Bronisz, Agnieszka & Murata, Yoshiyuki \\
\hline Brooks, James & Murayama, Takashi \\
\hline Bross, Peter & Muresan, Vlad \\
\hline Broszczak, Daniel & Murono, Shigeyuki \\
\hline Broussard, Gregory & Murotomi, Kazutoshi \\
\hline Brown, Euan R. & Murphy, Andrew \\
\hline Brown, James & Murphy, Paula \\
\hline Brown, Keith W. & Murray, Deirdre M. \\
\hline Brown, Robert J. & Murray, Rachael \\
\hline Brown, Stephen & Murray, Thomas Scot \\
\hline Browning, Glenn F. & Murthi, Padma \\
\hline Brugnera, Enrico & Muruve, Daniel \\
\hline Brullo, Chiara & Musch, Mark \\
\hline Brumeanu, Teodor-Doru & Mustafa, Ghazala \\
\hline Brummelte, Susanne & Mutti, Luciano \\
\hline Brumos Fuentes, Javier & Myers, Mark \\
\hline Brunasso, A. M. G. & Myers, Stephen \\
\hline Brunner, Andreas & Myllykallio, Hannu \\
\hline Bruns, Heiko & Myśliwiec, Hanna \\
\hline Bruschi, Fabrizio & Mysliwiec, Tami \\
\hline Brusslan, Judy A & Mystkowska, Joanna \\
\hline Brzozowski, Tomasz & Nagahashi, Masayuki \\
\hline Bubb, Kristen & Nagai, Katshito \\
\hline Bubici, Giovanni & Nagai, Katsura \\
\hline Bublitz, Margaret & Nagai, Kouhei \\
\hline Bubulya, Paula A. & Nagai, Noriaki \\
\hline Bucciantini, Monica & Nagai, Shigenori \\
\hline Buchet, Rene & Nagai, Yoshinori \\
\hline Buchheim, Mark & Nagaraj, Chandran \\
\hline Bucki, Robert & Nagaraja, Mamta Patel \\
\hline Buckley, Bradley A. & Nagarajan, Sureshbabu \\
\hline Buckley, M. & Nagaraju, G. P. \\
\hline Bucolo, Claudio & Nagata, Eiichiro \\
\hline Budak, Hikmet & Nagoya, Satoshi \\
\hline Buddaseth, Salma & Nagwekar, Janhavi \\
\hline Budka, Herbert & Nagy, Endre \\
\hline Buechler, Christa & Nagy, Istvan \\
\hline
\end{tabular}




\begin{tabular}{|c|c|}
\hline Buellesbach, Jan & Nagy, Laszlo \\
\hline Bueno, Marta & Nahashon, Samuel \\
\hline Buettner, Andreas & Nair, Vimal \\
\hline Bugge, Thomas & Najimi, Mustapha \\
\hline Bugla-Płoskońska, Gabriela & Nakagawa, Takuro \\
\hline Bugliani, Marco & Nakagawa, Yoshimi \\
\hline Bullon, Pedro & Nakahama, Ken-ichi \\
\hline Bultynck, Geert & Nakajima, Hiroko \\
\hline Bumgardner, Joel D. & Nakaki, Toshio \\
\hline Bum-Soo, Hahn & Nakamoto, Shingo \\
\hline Bunker, Alex & Nakamura, Masato \\
\hline Buonerba, Carlo & Nakamura, Nobuhiro \\
\hline Buono, P. & Nakamura, Shun-ichi \\
\hline Buonocore, Daniela & Nakamura, Tsukasa \\
\hline Burdo, Tricia H. & Nakanishi, Akira \\
\hline Burger, Michael & Nakano, Toshiaki \\
\hline Burgess, John A. & Nakashiro, Koh-Ichi \\
\hline Burghardt, Nesha & Nakayama, Yuji \\
\hline Burghardt, Robert & Nakchbandi, Inaam \\
\hline Burgstaller, Joerg & Nalivaeva, Natalia \\
\hline Burke, Mark & Nallasamy, Palanisamy \\
\hline Burow, Meike & Nalvarte, Ivan \\
\hline Burrin, Douglas G. & Nam, ONG Choon \\
\hline Burt, Tal & Namgaladze, Dmitry \\
\hline Burtea, Carmen & Nanavati, Charvi \\
\hline Burtey, Stéphane & Nanba, Daisuke \\
\hline Bus, James S. & Nandakumar, Kutty Selva \\
\hline Busardo, Francesco & Nandha Premnath, P. \\
\hline Buscarinu, Maria Chiara & Nandi, Saikat \\
\hline Busciglio, Jorge & Nandwana, Vikas \\
\hline Butler, Mark & Nanjappa, Manjunatha \\
\hline Butlin, Mark & Nanni, Loris \\
\hline Buttenschoen, Klaus & Napierala, Marek \\
\hline Butterfield, Russell J. & Napoletano, Chiara \\
\hline Büttner, Hartwig & Napoli, Pietro Emanuele \\
\hline Byun, Jonghoe & Naponelli, Valeria \\
\hline Caarls, Lotte & Narayanan, Anand \\
\hline Caballero-Garcia, Beatriz & Nardelli, Carmela \\
\hline Cabanillas, Beatriz & Nariai, Tadashi \\
\hline Cabral, Horacio & Narikawa, Rei \\
\hline Cabral, Jaydee & Narimatsu, Hiroto \\
\hline Cabrele, Chiara & Narkar, Vihang A. \\
\hline Cabrera-Fuentes, H. A. & Narute, Purushottam \\
\hline
\end{tabular}


Caburet, Sandrine

Caccamo, Daniela

Cacciatore, Ivana

Núñez De Cáceres González, F. F.

Cadamuro, Massimiliano

Cádiz Gurrea, M. D. L. L.

Caeiro, Maria

Caffarel, María Muñoz

Caffarelli, Carlo

Cafiero, Mauricio

Cahoon, Aubrey Bruce

Cai, Demin

Cai, Lu

Caiazzo, Elisabetta

Cairo, Gaetano

Cairrão, Elisa

Caivano, Antonella

Calabrese, Edward J.

Calado, Ângelo

Calder, Philip

Caldera, Fabrizio

Calì, Tito

Call, Mindy

Callaghan, Brid

Callendret, Benoit

Calorini, Lido

Calvano, Cosima D.

Calvo-Guirado, José Luis

Calzetta, Luigino

Calzone, Laurence

Camacho-Cristóbal, Juan J.

Camiciottoli, Gianna

Camire, Mary

Camlin, Nicole J.

Cammarata, Kirk

Campara, Maya

Campbell, Bradley

Campos, Eric

Campos, Narciso

Campuzano, Oscar

Cañas, Rafael A.

Candiani, Gabriele

Cândido, Elizabete
Narváez, Manuel

Nascimento, Kyria

Nass, Norbert

Nassuth, Annette

Nataf, Serge

Natarajan, Arutselvan

Natarajan, Sathish Kumar

Nault, Rance

Naumann, Todd A.

Naumova, Anna V.

Naumovski, Nenad

Navakauskiene, Ruta

Navarro, Esther Pérez

Naviglio, Silvio

Nawaz, Muhammad

Nazar, Ross N.

Nazaruk, Jolanta

Neely, Brian J.

Neergheen-Bhujun, Vidushi

Neilson, Andrew

Nejad, Mojgan

Nelson, Fred R. T.

Nepal, Madhav

Neubauer, Bernd A.

Neuhaus, Jochen

Neumann, Jake

Neureiter, Daniel

Neutelings, Godfrey

Nevšímalová, Soňa

Neymotin, Florence

$\mathrm{Ng}$, Dominic

Nguyen, Andrew V.

Nguyen, T. K. P.

$\mathrm{Ni}$, Hongmin

$\mathrm{Ni}$, Weiming

Nicholson, Louise F. B.

Nicolaides, Nicolas C.

Nicolaides, Theodore

Nicolas, Skuli

Nicoll, Rachel

Niculita-Hirzel, Hélène

Niederberger, Ellen

Niederman, Robert A. 
Cangkrama, Michael

Canini, Antonella

Cannavo, Elda

Cannone Falchetto, Augusto

Cansby, Emmelie

Cantalapiedra, Carlos P.

Cantile, Monica

Cao, Renzhi

Capdevila, Mercè

Capel, Carmen

Cappariello, Alfredo

Caprari, Patrizia

Carapelli, Antonio

Carare, Roxana Octavia

Carboni, Lucia

Cardoso, Susana

Cardoso, Susana M.

Cariati, Federica

Carlisi, Daniela

Carloni, Vinicio

Carlsen, Hanne Krage

Carmine, Guarino

Carmody, Ruaidhri

Carnevale, Daniela

Carol, Pierre

Carraro, Mauro

Carraro, Silvia

Carrasco-Pancorbo, Alegría

Carregal-Romero, Susana

Carretón, Elena

Carrier, Alice

Carrubba, Alessandra

Carson, Monica J.

Carter, Claire

Carter, Wayne

Cartland, Sian

Carulli, Lucia

Caruso, Giuseppe

Carvajal, Micaela

Carvalho, Katherine

Casal, Susana

Casalino, Elisabetta

CASARINI, Livio
Niedziela, Tomasz

Nielsen, Vance G.

Niemz, Angelika

Niessen, Frank B.

Nieto, Gema

Nieuwenhuizen, Niels J.

Nigris, Filomena De

Nijnik, Anastasia

Nikas, Spyros P.

Nikawa, Takeshi

Nikolaev, Sergey I.

Nikolakopoulou, Angeliki M.

Nilsson, Gunnar

Nilsson, Peter

Nilsson, Ulf J.

Nishiguchi, Masamichi

Nishimura, Kaneyasu

Nishimura, Norihisa

Nishimura, Taisuke

Nishiwaki, Hisashi

Nishiyama, Akira

Nisnevitch, Marina

Nissen, Jillian

Nistala, Ravi

Nitta, Takeshi

Nitulescu, George Mihai

Niu, Genhua

Nobili, Stefania

Nocentini, Giuseppe

Nociari, Marcelo

Nodomi, Seishiro

Nofer, Jerzy-Roch

Noge, Koji

Noghero, Alessio

Noguchi, Constance Tom

Noguchi, Eishi

Noguchi, Takuya

Noguera, Dan

Nohe, Anja

Nøhr, Mark K.

Nomikos, Tzortzis

Nomura, Mika

Nonami, Atsushi 
Cascio, Sandra

Casini, Giovanni

Casorati, Giulia

Castaldi, Alessandra

Castel, Hélène

Castella, Maria

Castellano, Orlando

Castiglioni, Bianca

Castle, Brian

Castorina, Alessandro

Castren, Eero

Caswell, Clayton C.

Cattaneo, Fabio

Catty, Patrice

Caudron-Herger, Maiwen

Cauli, Omar

Cava, Claudia

Cavada, Benildo Sousa

Cavalla, Paola

Cavallari, Marco

Cavani, Luciano

Cayot, Nathalie

Ceccarelli, Sara

Ceccherini Guicciardini, M. T.

Cecchetti, Serena

Cehofski, Lasse

Celli, Anna

Celli, Giovana

Cenci, Elio

Cengotitabengoa, Monica

Ceranowicz, Piotr

Cerchia, Laura

Ceresa, Brian

Ceriotti, Aldo

Cesare Marincola, Flaminia

Cesselli, Daniela

Chahrour, Maria

Chak, Kayam

Chakrapani, Anupam

Challacombe, Jean F.

Challagundla, Kishore B.

Challet, Etienne

Chamcheu, Jean Christopher
Nonappa, Nonappa

Nonhebel, Heather M.

Nookala, Suba

Noordermeer, Daan

Nørholm, Morten H. H.

Noris, Emanuela

Norman, Trevor

Norris, Erin

Norris, Gillian E.

Norton, Nadine

Nosoudi, Nasim

Novack, Gary D.

Novak, Richard

Novick, Daniela

Novío, Silvia

Nowak, Robert

Nowicki, Marcin

Nozu, Kandai

Núñez Garrote, José Ignacio

Nunn, Alistair V. W.

Nunomura, Wataru

Nuthikattu, Saivageethi

Nuutila, Kristo

Nyström, Kristina

O'neill, Catherine A.

Obara, Yutaro

Obermüller, Nicholas

Occhialini, Alessandro

Ochoa Corona, Francisco

Ocio, Enrique

O'Connell, Grace

Odenthal, Margarete

Odum, Niels

Offord, James

Ogasawara, Toru

Ogata, Tadanori

Ogi, Kazuhiro

Ogino, Shuji

Ogoh, Shigehiko

Ogunjirin, Adebowale

Oh, Keimei

Oh, Seikwan

Oh, Yoon Sin 
Chami, Belal

Chan, Ben C. L.

Chan, Chi Ho

Chan, Hue Sun

Chan, Julie

Chan, Wan

Chand, Sourabh

Chandaka, Giri Kumar

Chang, Chien-Chung

Chang, Chuang-Rung

Chang, Hui-hua

Chang, Ing-Feng

Chang, Jer-Ming

Chang, Jinho

Chang, Jungshan

Chang, K. C.

Chang, Kee-Lung

Chang, Ko-Tung

Chang, Long-Sen

Chang, Wei-Jen

Chang, Wen-Chi

Chang, Wen-Wei

Chao, Louis Kuoping

Chao, Wei-Ting

Chapel, Alain

Chapman, Mark A

Chapoval, Svetlana P.

Chari, Sudarshan

Charles, Luenda E.

Charlier, Edith

Charlton, Clivel

Chass, Gregory Adam

Chater, Caspar

Chatterjee, Jhinuk

Chatterjee, Ratna

Chatzigeorgiou, Antonios

Chaudhuri, Nazia

Chaurand, Pierre

Chaves-Martinez, F. J.

Chaves-Pozo, Elena

Checa, Antonio

Chegou, Novel N.

Chelbi, Sonia T.
O'Hagan, Heather M.

O'Hara, Steven

Ohe, Kenji

Ohkohchi, Nobuhiro

Ohmura, Koichiro

Ohnishi, Kohta

Ohnishi, Masatoshi

Ohno, Sho

Öhrfelt, Annika

Ohta, Yuko

Oikawa, Akira

Ojika, Makoto

Ojima, Koichi

Okada, Muneyoshi

Okada, Tomoyo

Okamoto, Koichi

Okamoto, Masami

Okamoto, Takayuki

Okino, Tatsufumi

Okleštková, Jana

Okochi, Mina

Okumura, Nobuo

Okumura, Tadayoshi

Olcese, James M.

Olejar, Kenneth J.

Oliveira, Paula Alexandra

Oliveira, Raquel

Oliveira, Rui

Oliveira-Ferrer, Leticia

Oliver, Brian

Oliver, Richard

Olivier, Elodie

Olivier, Gires

Olofsson Bagge, Roger

Olsen, Nancy

Olson, Michael W.

Oltra, Elisa

Omar, Syed Haris

Omranian, Nooshin

Ong, Eng Shi

Onyenwoke, Rob U.

Oon, Hazel H.

Oosterwijk, Egbert 
Chelmonska-Soyta, Anna

Chemat, Farid

Chen, Bor-Sen

Chen, Changhan

Chen, Chiachung

Chen, Chun-Chi

Chen, Chun-Jung

Chen, Chun-Lin

Chen, Gen-Hung

Chen, Guoxun

Chen, Hongwei

Chen, Hui-Wen

Chen, Jiann-Chu

Chen, Jianwu

Chen, Jiao

Chen, Kai-Yi

Chen, Kevin

Chen, Kun-Ming

Chen, Lei-Shih

Chen, Linyi

Chen, Miaw-Ling

Chen, Min-Huey

Chen, Po-Yuan

Chen, Rong-Jane

Chen, Ruei-ming

Chen, Suzie

Chen, Tianbao

Chen, Tong

Chen, Tzong-Yueh

Chen, Wayne

Chen, Wei-Yu

Chen, Yei-Tsung

Chen, Yeong-Ren

Chen, Yiliang

Chen, Yu-Chih

Chen, Zhe-Sheng (Jason)

Chen, Zhihang

Chen, Zhijie

Chen, Zhonghua

Chénais, Benoît

Cheng, Jia

Cheng, Juei-Tang

Cheng, Xinlai
Oosting, Jan

Opazo, Felipe

Oppert, Brenda

Oppido, Piero Andrea

Oprian, Daniel

Orban, Laszlo

Ordas, Bernardo

Orecchio, Santino

Orecchioni, Marco

Orengo, Dorcas

Orlova, Elena V.

Ornstein, Moshe C.

Orosa, Beatriz

Orozco, Luis

Ortega, Natividad

Orth, James D.

Ortolano, Saida

Ory, Stéphane

Orynbayeva, Zulfiya

Orzechowski, Arkadiusz

Osborn, Mark

O'shaughnessy, K. M.

Oshima, Yoshimi

Oshima, Yusuke

Oshiumi, Hiroyuki

Osier, Nicole

Osman, Narin

Osolodkin, Dmitry

Osorio, E. Yaneth

Osorio, Johan

Osses, Nelson

Otani, Keisuke

Othumpangat, Sreekumar

O'Toole, Sharon A.

Otsuka, Fuminori

Otsuka, Yuzuru

Ouazzani, Jamal

Oury, Cécile

Owen, Shawn

Oyama, Fumitaka

Oyoshi, Takanori

Ozaita Mintegui, Andrés

Ozaki, Iwata 
Cheng, Yu-Jung

Chengwen, Sun

Cheon, Choong-ill

Cherkaoui Malki, Mustapha

Cherniack, Evan Paul

Cherry, Jonathan

Chi, Chia-Yu

Chi, Ching-Shiang

Chiadò, Alessandro

Chiang, Hsiu-Mei

Chiang, Ming-Ko

Chiang, Tai-an

Chiaraluce, Roberta

Chiariello, Mario

Chiba, Yoshihiko

Chidambareswarapattar, Chakkaravarthy

Chien, Chihyen

Chien, Hung-Yu

Chiessi, Ester

Chilian, William M.

Chilosi, Marco

Chim, Stephen

Chiou, Ya-Ling

Chirumbolo, Salvatore

Chitarra, Walter

Chiu, Ka Fung Peter

Chiu, Tsai-Hsin

Chlichlia, Katerina

Chmurzynska, Agata

Cho, Dong-Woo

Cho, Joo-Youn

Cho, Seung Hak

Cho, Ssang-Goo

Cho, Yoon Hee

Chobot, Vladimir

Choi, Dae Eun

Choi, Huimahn A.

Choi, Je-Min

Choi, Je-Yong

Choi, Seong-Ho

Choi, Woonyoung

Cholkar, Kishore

Chong, Lee-Gau
Ozaki, Toshinori

Özalp, V. C.

Ozeki, Yasuhiro

Ozkok, Abdullah

Ozsvari, Bela

Pace, Betty S.

Pacella, Elena

Pacheco, Mário

Paci, Maurizio

Pacios, Luis F.

Paduch, Roman

Padula, Andrew

Padula, Matthew

Pae, Eung-Kwon

Paek, Andrew

Pagani, Franco

Pagani, Stefania

Pagano, Luca

Pagano, Maria

Paggetti, Jérôme

Paggiaro, Pierluigi

Pagni, Fabio

Paik, Jisun

Paik, Yong-Han

Pain, Bertrand

Paíno, Teresa

Paiva, Cintia S. De

Pajak, Paulina

Pal, Rituraj

Palacios, Oscar

Palanisamy, Satheesh Kumar

Palei, Ana

Palese, Peter

Palitti, Fabrizio

Paliyath, Gopinadhan

Pallotta, Isabella

Palmero, Daniel

Palmieri, Dario

Palmioli, Alessandro

Palumbo, Carla

Paluzzi, Jean-Paul V.

Pan, Hung-Chuan

Pan, Miao 
Chou, Cheng-Yang

Chou, Kuo-Chen

Choudhary, Sanjeev

Choudhury, Devasmita

Choudhury, Mahua

Choudhury, Malay

Chouker, Alexander

Chowdhry, Sudhir

Christ, Torsten

Christensen, Brock

Christians, Elisabeth

Christians, Julian

Christians, Kathleen K.

Christodoulou, Maria-Ioanna

Christoffersen, Mette

Christopher W., Kirby

Chruszcz, Maksymilian

Chrzanowski, Łukasz

Chu, Shidong

Chua, Anita C. G.

Chua, Melvin

Chuang, Lea-Yea

Chuang, Yung-Jen

Chüeh, Anderly C.

Chui, Helena

Chun, Heung Jae

Chun, Kyung-Soo

Chung, Byung Min

Chung, Donghoon

Chung, Jayong

Chung, Jong Hoon

Chung, Sungjin

Chyau, Charng-Cherng

Ciaccio, Marcello

Ciancetta, Antonella

Ciapetti, Gabriela

Ciavattini, Andrea

Cicala, Michele

Cicciu, Marco

Ciccone, Marco Matteo

Cicero, Arrigo

Cichero, Elena

Cichorek, Miroslawa
Panagiotidis, Mihalis

Panagiotopoulos, Ioannis A.

Panaro, Maria Antonietta

Pandey, Avinash Chandra

Pandey, Girdhar K.

Pandita, Tej

Panfoli, Isabella

Pang, Jinjiang

Pani, Bibhusita

Panicker, Rajesh

Panico, Antonio

Panov, Konstantin

Panzella, Lucia

Paoli, Paolo

Papaccio, Gianpaolo

Papadimitriou, Lampros

Papadimitriou, Sofia A.

Papadimitriuou, K.

Papaioannou, Dionissios

Papaleo, Francesco

Papantonis, Argyris

Papazafiropoulou, A. K.

Papenfort, Kai

Papet, Isabelle

Papetti, Adele

Papi, Alessio

Papini, Alessio

Papp, András

Pappalardo, Daniela

Paradowska-Gorycka, Agnieszka

Parashar, Deepak

Paraskevis, Dimitrios

Parayath, Neha N.

Parcells, Mark

Pardal, Ricardo

Pardigon, Nathalie

Parekh, Dhruv

PARINE, NARASIMHA REDDY

Parisi, Cristian

Parisini, Emilio

Park, Byung

Park, Chankyu

Park, Hae Ryoun 
Cignarella, Andrea

Cimmino, Amelia

Cindrova-Davies, Tereza

Cinelli, Patrizia

Ciobica, Alin

Cipolla, David

Cippitelli, Marco

Cirilli, Marco

Cirillo, Emilia

Cisowsky, Jaroslaw

Citro, Vale

Clark, Michelle A.

Clarkson, Andrew

Clausen, Frederik

Cleary, Margot P.

Clément, Yves

Clementi, Nicola

Cleren, Carine

Clericuzio, Marco

Clevenger, Josh

Cloutier, Conrad

Coan, P. M.

Cobley, James

Coccheri, Sergio

Cocco, Cristina

Cocetta, Giacomo

Cochran, Blake

Cohen, Dan

Cohick, Wendie

Cohn, Martin

Colecchia, David

Coleman, William B.

Coley, Helen M.

Collett, Andrew

Collier, Jason

Collin, Rob

Collins, Maurice N.

Cologna, Stephanie

Colombi, Marina

Colombo, Emanuela

Colonna, Giovanni

Colquhoun, Thomas A.

Colussi, GianLuca
Park, Hyun-Woo

Park, Joshua

Park, Junsoo

Park, Maiyon

Park, Sam-Yong

Park, Sung-Gyoo

Parker, Caroline

Parker, Tony J

Parnetti, Lucilla

Parola, A. Jorge

Parrish, Alan

Parsons, Jason L.

Partch, Carrie L.

Parthasarathy, Srinivas

Parveen, Iffat

Parys, Jan B.

Pascale, Alberto

Pascale, Rosa

Pascarelli, Nicola Antonio

Pascu, Irina

Pasini, Luigi

Paslakis, Georgios

Pasqualoto, Kerly

Passamonti, Sabina

Passarinha, L. A. P.

Pastor, Fernando

Pastor, Jesús

Pastores, Gregory M.

Pasz, Lidia Sas

Paszkiewicz, Sandra

Patel, Daxesh P.

Patenaude, Mathew

Pathakoti, Kavitha

Patil, Naeem K.

Patil, Rajkumar V.

Patrice, Thierry

Pattekari, Pravin

Patterson, Jennifer

Paul, Antoni

Paul, Matthew

Paulus, Werner

Pavagadhi, Shruti

Pavankumar, Theetha 
Comai, Stefano

Comfort, K. K.

Commisso, Mauro

Condorelli, Daniele Filippo

Conese, Massimo

Conlon, John Michael

Connaughton, Victoria P.

Conti, Heather

Conti, Lucio

Contino, Marialessandra

Contreras-Moreira, Bruno

Cooke, Ira R.

Cooke, Marcus

Cook-Mills, Joan M

Coppedè, Fabio

Coppotelli, Giuseppe

Corbetta, Sabrina

Cordo Russo, Rosalia I.

Cordoba-Diaz, D.

Corella, Dolores

Corniola, Rikki

Correia-de-Sá, Paulo

Cortés-Castell, Ernesto

Cortese, Katia

Cortez, Maria Angelica

Costa, Maria Manuela

Costantini, Susan

Costanzo, Francesco

Costa-Rodrigues, João

Cotella, Diego

Cotman, Susan

Courville, Elizabeth L.

Cousin, Xavier

Coutinho, João Paulo

Coutinho, Teresa

Covello, Giuseppina

Covès, Jacques

Cowell, Ian

Coyle, Beth

Coyle, Patricia K

Craggs, Timothy D.

Cran, Marlene J.

Crawford, Bryan D.
Pavlidis, Ioannis

Pavlik, Edward J.

Pavlov, Tengis

Pawar, Snehalata

Pawełkowicz, M. E.

Pawlak, Agnieszka

Payne, Annette

Pazdro, Robert

Peana, Alessandra T.

Peana, Massimiliano F.

Pearson, Mark

Pechanova, Olga

Pécheur, Eve-Isabelle

Pedersen, Anders

Pedeux, Rémy

Pedotti, Rosetta

Pedraza-Chaverri, José

Peeples, Mark

Peffers, Mandy J.

Pegolo, Sara

Peijnenburg, Willie

Peinado, Juan R.

Peiris, Madusha

Peitzsch, Claudia

Pejin, Boris

Pekin, Deniz

Pelagalli, Alessandra

Pellegrini, Gretel G.

Pelletier, Guillaume

Pellis, Alessandro

Pelosi, Paolo

Peluso, Gianfranco

Peluso, Ilaria

Pemov, Alexander

Peña, Maria Marjorette O.

Pendyala, Gurudutt

Penfornis, Patrice

Peng, Chien-fang

Peng, Lifeng

Peng, Robert

Peng, Teng

Peng, Wen-Huang

Penissi, Alicia B. 
Crawford, Gemma

Creamer, Rebecca

Crescenzi, Anna

Crescioli, Clara

Crespan, Emmanuele

Crespi, Martin

Crespo, Mariano Sánchez

Croci, Diego

Croset, Martine

Cross, Carroll E.

Crotti, Tania

Cruz, Celia

Cruz-Fuentes, Carlos S.

Csabafi, Krisztina

Csányi, Gábor

Cubellis, Maria

Cubero, Francisco Javier

Cucchiarini, Magali

Cudalbu, Cristina

Cuesta, Alberto

Cui, Haitao

Cui, Xiaoying

Cui, Yi

Cullen, Carlie

Culotta, Valeria Cizewski

Culty, Martine

Cuneo, Matthew J.

Cunningham, Michael

Cunningham, Patrick

Curci, Pasquale Luca

Curtis, Nicholas

Cusanelli, Emilio

Czabanka, Marcus

Czarnocka, Weronika

Czeglédi, Levente

Czerwinska, Monika

Czikora, Istvan

Czubryt, Michael P.

Da Silva Filho, Ademar Alves

Dabrowiak, James

Dacheux, Jean-Louis

Daftarian, Pirouz

Dai, Yang D
Penn, Linda

Pennington, Kathleen

Pennock, Nathan

Pennypacker, Keith

Pensabene, Virginia

Peracaula, Rosa

Perego, Carla

Pereira, C.

Pereira, David

Pereira, Joana L.

Perera, Omaththage

Perera, Rushika

Perez, Juan J.

Pérez-Camino, Mari Carmen

Pérez-Castrillón, José L.

Perez-Gracia, Teresa

Pérez-Pérez, José Manuel

Perez-Stable, Carlos

Pergament, Eugene

Peris, Ketty

Perrais, Michaël

Perreault, Jonathan

Perrella, Giorgio

Perrine-Walker, Francine M.

Perrotti, Nicola

Perry, Christopher G. R.

Perticone, Francesco

Pes, Giovanni Mario

Pešić, Milica

Pesquet, Edouard

Peterbauer, Clemens Karl

Petering, David

Peters, Eva Milena Johanne

Petersen, Björn

Peterson, Julia

Petit, Patrice X.

Petrescu, Anca D.

Petrini, Stefano

Petropoulos, Isabelle

Petrova, Olga E.

Petruczynik, Anna

Petrzik, Karel

Petta, Ioanna 
Dai, Yun

Dailianis, Stefanos

Daina, Antoine

Dale, Russell

D'Alessandro, Angelo

Dalli, Jesmond

Damaraju, Sambasivarao

Damjanovski, Sashko

Da'na, Enshirah

D'Andrea, Antonio

D'Angiolella, Vincenzo

Daniel, Marie-Christine

Danila, Daniel C.

Danilenko, Michael

Dansette, Patrick M.

Darby, Ian

D'Argenio, Valeria

Dark, Michael J.

Darwish, Omar

Das, Anindita

Das, Lalitendu

Das, Undurti

Das, Viswanath

Dasgupta, Subham

Datta, Rupsa

Daugrois, Jean-Heinrich

D'Auria, Maria Valeria

Dave, Sandeep

Daver, Naval

Daverey, Amita

Davide, Francomano

Davidson, Cristin

Davidson, Doug

Davies, Jeffrey S.

Davis, Frank

Davis, Michael E.

Davis, Michael J.

Dawn, Arnab

Dawood, Mahmoud A. O.

De Aberasturi, D. J.

De Almeida Coelho De Sousa, Maria João

De Amicis, Francesca

De Andrés Cara, Damián F.
Pettit, Allison

Petukh, Marharyta

Pezzuto, Aldo

Phatarpekar, Prasad

Phelps, Patricia E.

Philip, Molyneaux

Philipp, Stephan

Philips, Neena

Phillips, Jacqueline

Phipps, Simon

Picardi, Ernesto

Picardo, Mauro

Picariello, Gianluca

Piccoli, G. B.

PICOT, Cedric

Picozzi, Mario

Piedade, Ana Paula

Piedra, Pedro A.

Piiper, Albrecht

Pilati, Stefania

Pilaz, Louis-Jan

Pillay, Viness

Pilon-Thomas, Shari

Pilot, Guillaume

Pina, Melanie

Pina, Sandra

Pineda, Asun Monfort

Pineda, Miguel

Pinho, Brígida Ribeiro

Pintea, Adela

Pinto, Antonio

Pinto, Diana C. G. A.

Pinto, Mafalda

Pinton, Paolo

Pinzani, Pamela

Piomboni, Paola

Piplani, Honit

Pires, Carla

Pirici, Daniel

Pirillo, Angela

Piróg, Katarzyna A.

Piskuła, Mariusz K.

Pisoschi, Aurelia Magdalena 
De Aza, Piedad

De Berardis, Domenico

De Biase, Dario

De Boer, M.K. (Marrit)

De Bortoli, Michele

De Brevern, Alexandre

De Bruyne, Elke

De Deurwaerdère, Philippe

De Feo, Vincenzo

De Francesco, Francesco

De Geest, Bart

De Gonzalo, Gonzalo

De Gonzalo-Calvo, David

De Graaf, Petra

De Jong, Jill L.O.

De La Cruz, José Pedro

De La Fuente, Hortensia

De La Garza, Mireya

De La Luz Garcia-Hernandez, Maria

De La Monte, Suzanne

De La Serrana, Daniel Garcia

De Lago, Eva

De Lange, Pieter

De Lourdes Pereira, Maria

De Luca, Luciana

De Maagd, Ruud

De Martin, Rainer

De Mattei, Monica

De Miguel-Beriain, Iñigo

De Paepe, Boel

De Paolis, Angelo

De Pergola, Giovanni

De Re, Valli

De Rosa, Maria Cristina

De Rossi, Anita

De Spiegelaere, Ward

De Vaufleury, Annette

De Vos, John

De Vrieze, Erik

De Wever, Olivier

De Yoreo, Jim

De, Kuntal

Dean, Afshan
Pistone, Alessandro

Pitt, Samantha

Pitto, Letizia

Piva, Terrence

Pivac, Nela

Piwowar, Agnieszka

Pizzolanti, Giuseppe

Pla, Davinia

Placido, Jersson

Plantier, Laurent

Plarre, Ruediger

Platania, Chiara

Platé, Manuela

Plaza-Díaz, Julio

Plaza-Zabala, Ainhoa

Plażuk, Damian

Plazzi, Federico

Pleskot, Roman

Pluquet, Olivier

Pniewski, Tomasz

Pocheć, Ewa

Podechard, Normand

Poeggeler, Burkhard H.

Poggi, Alessandro

Pognonec, Philippe

Poidatz, Dorothée

Poitz, David M.

Pokora, Wojciech

Pokrywka, Andrzej

Polacco, Joseph C.

Polaina, Julio

Polakovičová, Mája

Poldermans, Don

Poleszak, Ewa

Poltronieri, Palmiro

Pompili, Maurizio

Ponomarenko, Julia

Ponomarev, Eugene

Ponsaerts, Peter

Poole, Kate

Pooler, Darcy

Pop, Oana

Popa-Wagner, Aurel 
Dean, Justin

Deb, Subrata

DeCicco-Skinner, Katie

Dedecker, Peter

Deevska, Gergana

Degano, Ilaria

Degrassi, Francesca

Dei Giudici, Silvia

De-Juan-Pardo, Elena M.

Dekaban, Gregory A.

Dekker, Marloes

Del Gaudio, Costantino

Del Hierro, Isabel

Del Mistro, Annarosa

Del Portillo, Armando

Del Pozo, Olga

Del Re, Brunella

Del Real, Gustavo

Delannoy, Philippe

Deleidi, Michela

Delhanty, Patric J. D.

Delihas, Nicholas

D'elios, Mario M.

Dell'Agli, Mario

Dellis, Olivier

Delogu, Giovanna

Delorme, Vincent

Delporte, Christine

Demir, Fatih

Demir, Ihsan Ekin

Demyanets, Svitlana

Den Hartigh, Laura J.

Deng, Peng

Deng, Qing

Deng, Xufang

Deng, Zhanao

Denizot, Benoit

Denovan-Wright, Eileen M.

Deriu, Marco A.

DeRosa, Maria

Desai, Abhishek

Desai, Jigar

Desando, Giovanna
Popović Đorđević, Jelena

Porebski, Grzegorz

Porter, Craig

Portis, Ezio

Poschenrieder, Charlotte

Possidente, Bernard

Potenza, Nicoletta

Potiron, Vincent

Poulou, Myrto

Powell, Ann L. T.

Powell, Daniel

Powell, Joshua

Powers, Jennifer

Pozo, Óscar J.

Prado, Raquel

Prante, Olaf

Prasad, Asuri N.

Prats, Anne-catherine

Preciado, Diego

Preissner, Klaus T.

Prensner, John

Prentice, Howard

Prieto, Isabel

Prieto-Lloret, Jesus

Primiceri, Elisabetta

Prince, Jose

Prinsi, Bhakti

Prinz, Jörg C.

Proctor, Carole

Proestos, Charalampos

Professor Siu, Ming Fai

Profirovic, Jasmina

Proft, Thomas

Profumo, Elisabetta

Prohens, Jaime

Prokai, Laszlo

Proost, Paul

Proudfoot, Amanda E. I.

Pruett, Drew

Pruna, Alina

Przekora, Agata

Przepiera-Będzak, Hanna

Przyborowski, Jerzy 
Deshmukh, Rupesh

D'Este, Matteo

Dettweiler, Ulrich

Devan, Bryan D.

Devasundaram, Santhi

Devesa, Jesus

Devine, Patrick J.

Devriendt, Bert

Devy, Jérôme

Dewitte, Heleen

Dhanasekaran, Danny N.

Dhanasekaran, Saravana M.

Dhoot, G. K.

Di Daniele, Nicola

Di Fazio, Pietro

Di Giacomo, Silvia

Di Guglielmo, Gianni M.

Di Liegro, Italia

Di Marco, Stefano

Di Masi, Alessandra

Di Nardo, Matteo

Di Primo, Carmelo

Di Renzo, L

Diack, Abigail B.

Diana, Patrizia

Diapari, Marwan

Díaz, Isabel

Diaz, Jose Antonio

Díaz, José Fernando

Dickson, Suzanne

Didonna, Alessandro

Diekman, Alan

Diermeier, Sarah

Dierssen, Mara

Díez, David

Dijkhuizen, Lubbert

Dijkstra, Gerard

Dikalov, Sergey

Dimitrakopoulou-Strauss, A.

Dimitrios, Stagos

Dimitrov, Kiril M.

Dimova, Elitsa

Ding, Qiang
Psarra, Anna-Maria G.

$\mathrm{Pu}$, Jing

Pucciarelli, Sandra

Puccio, Ava

Puchta, Holger

Pugh, Nicholas

Puiggalí, J.

Pulignani, Silvia

Pulinilkunnil, Thomas

Puri, Akshit

Püschel, Gerhard P.

Pushkar, Yulia

Pusztaszeri, Marc

Putz, Mihai

Puvvula, Pavan Kumar

Pytka, Karolina

Qi, Dake

Qi, Robert Z.

Qi, Rongsu

Qi, Xin

Qian, Hong

Qian, Li

Qian, Pengxu

Qin, Gangjian

Qin, Helena

Qin, Hongwei

Quattrocelli, Mattia

Quémerais, Bernadette

Quesada Pérez, Víctor Manuel

Quigley, Eamon

Quillard, Thibaut

Quintana, José

Quock, Ryan

Qvit, Nir

Rabbani, Naila

Rabien, Anja

Rabouille, Catherine

Rachek, Lyudmila

Rachmawati, Dessy

Radeke, Monte J.

Radenkovic, Miroslav

Rafikova, Olga

Raftery, Rosanne 
Ding, Wen-Xing

Dings, Ruud P.M.

Dinh, Dzung H.

Dini, Luciana

Dinischiotu, Anca

Diniz, Carmen

Dioum, Elhadji M.

Distel, Martin

Dittmer, Neal T.

Ditzel, Mark

Dixon, Ian M.C.

Dmitriev, Oleg

Do Canto, Javier

Do, Sun Hee

Dobens, Leonard L.

Dobrowolska, Grażyna

Docheva, Denitsa

Doddapaneni, Ravi

Doekes, Gert

Doi, Kazuya

Doisneau-Sixou, Sophie

Dolei, Antonina

Dolinsky, Vernon W.

Dolo, Vincenza

Domingos, Pedro M.

Domingues, Fernanda

Domingues, Renan B.

Domínguez-Álvarez, Enrique

Domozych, David S.

Donadio, Carlo

Donato, Rosario

Dong, Feng

Dong, Mingdong

Dong, Zheng

Donnelly, Oliver

Donnini, Sandra

Donohue, Kevin

Donzelli, Elisabetta

Dores, Michael R.

Dorfman, Damian

Dormond, Olivier

Dorrance, Anne M.

D'Orsi, Beatrice
Raggi, Lorenzo

Ragusa, Maria Antonietta

Rahe, Michael C.

Rahimi, Farid

Rahman, Milladur

Rai, Prakash

Raimondi, Lavinia

Raimondo, Stefania

Rainer, Peter $\mathrm{P}$

Rainero, Elena

Raingeaud, Joël

Raj, Madhwa H. G.

Rajaram, Murugesan

Rajendran, Ranjith Kumar

Rajendran, Vazhaikkurichi

Rajgor, Dipen

Rajnarayanan, Rajendram

Rajpurohit, Surendra

Raju, Jayadev

Rakers, Christin

Rakitin, Aleksei

Ralli, Massimo

Rallis, Micael

Ram Kumar, Ram Mohan

Ramalingam, Naveen

Ramalingam, Vetrivelan

Ramani, Komal

Ramani, Vijay

Ramaraj, Pandurangan

Ramasamy, Srinivasan

Ramchandani, Divya

Ramil, Carlo

Ramírez, Daniel Robledo

Ramírez, Víctor

Ramonda, Roberta

Ramos, Sonia

Rampias, Theodoros

Ramsahoye, Bernard

Ramsay, Alan

Ramsbottom, Simon

Ramshaw, Hayley

Ran, Chongzhao

Rana, Dipak 


\begin{tabular}{|c|c|}
\hline Dosoky, Noura S. & Randall, Stephen K. \\
\hline Dossena, Silvia & Rangachari, Manu \\
\hline Doulgeraki, Agapi & Ranjan, Alok \\
\hline Doumbia, Seydou & Ranjit, Suman \\
\hline Douros, Jonathan D. & Ranney, Thomas G. \\
\hline Dovinova, Ima & Rantamaki, Tomi \\
\hline Dovizio, Melania & Ranzato, Elia \\
\hline Doyle, Siamsa & Rao, C. V. \\
\hline Draeger, Annette & Rapacz, Marcin \\
\hline Draijer, Richard & Raposio, Edoardo \\
\hline Drenjančević, Ines & Rapp, Alexander \\
\hline Drews, Oliver & Räsänen, Leena A. \\
\hline Driscoll, James J & Rasid, Orhan \\
\hline Drobizhev, Mikhail & Rasinger, Josef Daniel \\
\hline Drougard, Anne & Raspanti, Mario \\
\hline Drysdale, Thomas & Rastegar, Mojgan \\
\hline Du, Min & Ratajewski, Marcin \\
\hline Duarte, Fátima & Ratcliffe, Ian \\
\hline Dubey, Ashish & Raterman, H. G. \\
\hline Dubey, Ramin & Rathinasabapathy, A. \\
\hline Dubielecka, Patrycja M. & Ratkowsky, David A. \\
\hline Dubos, Christian & Ratovitski, Edward \\
\hline Dubrova, Yuri & Ratz-Łyko, Anna \\
\hline Ducza, Eszter & Rauch, Bernhard \\
\hline Dudás, József & Raupach, Michael \\
\hline Dudeja, Pradeep & Rauz, Saaeha \\
\hline Dudok, Jeroen & Ravaioli, Stefano \\
\hline Duennwald, Martin L & Ravegnini, Gloria \\
\hline Duffy, Aaron M. & Ravet, Karl \\
\hline Dugé De Bernonville, Thomas & Ravuri, Sudheer \\
\hline Duh, Pin-Der & Rawal, Aditya \\
\hline Duke, Stephen O. & Rawashdeh, Oliver \\
\hline Duman-Scheel, Molly & Rawel, Harshadrai \\
\hline Dumic, Jerka & Rawlings, Neil \\
\hline Dumke, Roger & Ray, Jayashree \\
\hline Dumont, Patrick & Ray, Udayan \\
\hline Dunbar, Gary L. & Rayan, Anwar \\
\hline Dunn, Louise & Raychaudhuri, Siba P. \\
\hline Duong, Hai M. & Reader, Jocelyn C. \\
\hline Duong-Quy, Sy & Reale, Marcella \\
\hline Duperray, Alain & Rebmann, Vera \\
\hline Duque, Paula & Recchia, Alessandra \\
\hline Durazzo, Alessandra & Reddy, Sakamuri \\
\hline
\end{tabular}




\begin{tabular}{|c|c|}
\hline Duressa, Dechassa & Redell, Michele \\
\hline Durgo, Ksenija & Redmer, Torben \\
\hline Durlik, Magdalena & Redondo Muñoz, Javier \\
\hline Durnford, Dion & Reese, R. Neil \\
\hline Duryee, Michael J. & Reeve, Amy \\
\hline Dusi, Veronica & Reeve, Vivienne \\
\hline Dussert, Stéphane & Regal, Jean \\
\hline Dutta, Arijit & Regalado-González, Carlos \\
\hline Dutta, Palash & Reginato, Mauricio J. \\
\hline Duverger, Eric & Regnard, Jean-Luc \\
\hline Dvorakova-Hortova, Katerina & Rehrig, Erin M. \\
\hline Dyr, Jan & Reid, Christopher W. \\
\hline Dziedzic, Arkadiusz & Reidy, Paul \\
\hline Dziga, Dariusz & Reigosa, Manuel J. \\
\hline Dziubla, Thomas & Reilly, Karlyne M. \\
\hline E. Katerinopoulos, H. & Reinach, Peter S. \\
\hline Easmon, Johnny & Reinders, Joerg \\
\hline Eben, Astrid & Reinders, Yvonne \\
\hline Ebo, Didier & Reis, Henning \\
\hline Ebrahimi, K. H. & Reiser, Peter J. \\
\hline Ebrahimi-Fakhari, Darius & Reisinger, Nicole \\
\hline Echevarria, Miriam & Reisman, Scott A. \\
\hline Eckardstein, Arnold & Reljic, Rajko \\
\hline Eckardt, Kristin & Relógio, Angela \\
\hline Ecke, Thorsten & Remaley, Alan T. \\
\hline Eckhard, Ulrich & Remaut, Katrien \\
\hline Edamakanti, Chandrakanth & Remesh, Soumya G. \\
\hline Eder, Iris E. & Remuzzi, Giuseppe \\
\hline Eder, Klaus & Ren, Jianguo \\
\hline Edin, Nina Jeppesen & Renaudineau, Yves \\
\hline Edinson, Yara-Varón & Renes, Johan \\
\hline Edirisinghe, Mohan & Renfroe, Michael \\
\hline Edwards, Joshua R. & Renneckar, Scott \\
\hline Edwards, Marten John & Renò, Filippo \\
\hline Eferl, Robert & Renovanz, Mirjam \\
\hline Efird, Jimmy & Revelli, Luca \\
\hline Egashira, Nobuaki & Revilla, Pedro \\
\hline Egawa, Gyohei & Reymond, Jean-Louis \\
\hline Egerszegi, István & Reynolds, Lindsay M. \\
\hline Eggeling, Lothar & Rezaei-Ghaleh, Nasrollah \\
\hline Eggink, Dirk & Rezvani, Khosrow \\
\hline Ehlen, J. Christopher & Rialland, Mickaël \\
\hline Ehninger, Armin & Riazi, Sheila \\
\hline
\end{tabular}


Ehrich, Marion

Eichhorn, Lars

Eide, David

Eidenberger, Thomas

Eiroa, José Luis

El Midaoui, Adil

El Muayed, Malek

El-Ali, Ayman

Elbaum, Rivka

Elbayoumi, Tamer

Elble, Randolph C.

Eldarov, M. A.

Eleftheriadis, Theodoros

El-Habr, Elias A.

Elhanafi, Ahmad Omar

El-Heliebi, Amin

Elibox, Winston

Eliseev, Roman A.

El-Kadi, Samer

El-kereamy, Ashraf

Ellervik, Christina

Elleuche, Skander

Elli, Stefano

Elliott, Paul R.

El-Matbouli, Mansour

Else, Kathryn

Elshimali, Yahya I.

Emery, Peter

Emiliani, Carla

Emoto, Makoto

Empl, Michael

Emri, Eszter

Endo, Tatsuro

Engel, Paul C.

Engelhart, Aaron

Engh, Richard A.

Engineer, Crystal T.

Enguita, Francisco

Enugutti, Balaji

Eppler, Elisabeth

Erban, Tomas

Erdődi, Ferenc

Erenpreisa, Jekaterina
Ribeiro, Edna

Ribeiro, Isabel

Ribeiro, Maria Henriques L.

Ribeiro-Barros, Ana I.

Riccetti, Laura

Rice, Lauren J.

Rich, Jeremy N.

Richa, Tambi

Richards, Renée Stirling

Richardson, Alan

Richetto, Juliet

Richly, Holger

Ricotti, Leonardo

Rieder, Michael

Riediger, Thomas

Rieger-Christ, Kimberly M.

Rienzo, Valentina Di

Riga, Maria

Rigante, Donato

Rigas, Stamatis

Rigó, János

Rijt, Leonie Van

Rikke, Norregaard

Riley, David G.

Rimbach, Gerald

Rimoldi, Luca

Rimoldi, Simona

Rimondini, Lia

Rinnerthaler, Gabriel

Rinnerthaler, Mark

Risatti, Guillermo

Rishi, Arun

Risk, Janet M.

Ristori, Giovanni P.

Rivera, Victor

Rivero, Francisco

Rivero, Rosa

Riveron, Jacob M.

Rivero-Pérez, M. Dolores

Rizzo, Angela Maria

Rizzo, Roberta

Rizzolio, F.

Rizzuti, Bruno 
Eri, Raj

Eritja, Ramon

Ernst, Robert K.

Escames, Germaine

Escobar, Carolina

Eskandari, Arvin

Espada, Jesús

Espen, Luca

Espinosa-Diez, Cristina

Esposito, Ciro

Esposito, Debora

Esposito, Emilio Xavier

Esposito, Sergio

Estaras, Concepcion

Esterio, Marcela

Esteve-Romero, Josep

Estevez, Jorge D.

Estevinho, Leticia

Etienne, Becht

Etxebeste, Oier

Euaggelos, Gikas

Euler, Gerhild

Evans, Franklin

Evans, Gareth John Owen

Evans, Gethin

Evans, Joseph L.

Evans, Malkanthi

Eve, David J.

Everett, Thomas

Ezendam, Janine

Ezura, Yoichi

F Wallace, Daniel

Fabiani, R.

Fabris, Linda

Fabris, Luca

Facchini, Gaetano

Faehling, Martin

Faenza, Irene

Fairweather, Stephen

Faísca, Patrícia

Falcieri, Elisabetta

Falkinham, Joseph

Famiani, Franco
Roan, Nadia R.

Robert, Eric

Robert, Jerome

Robert, Leema

Robert-McComb, Jacalyn J.

Robinson, Clive

Robinson, Karen

Robles Ramos, Pedro

Robson, Geoff

Robson, Matthew

Roccheri, Maria Carmela

Rocha Ferreira, Eridan

Rocha, Eduardo

Rocha, Sonia

Rochaix, Jean-david

Roche, Daniel Barry

Rödel, Franz

Rodella, Luigi F.

Rodenbeck, Stacey Dineen

Rodius, François

Rodrigo, Ismael

Rodrigo, María Jesús

Rodrigues, Alírio

Rodrigues, Melanie

Rodrigues, Nuno

Rodrigues, Olivier

Rodríguez Romero, Adela

Rodriguez, Alejandro

Rodríguez, Alexander J.

Rodriguez, Christophe

Rodriguez-Aguayo, Cristian

Rodriguez-Algaba, Julian

Rodriguez-Lafrasse, Claire

Rodríguez-Llorente, I. D.

Rodriguez-Menocal, Luis

Roe, R. Michael

Roeb, Elke

Roelen, Bernard

Roelofs, Jeroen

Roffi, Alice

Rogozhin, Eugene

Rogozin, Igor

Roh, Changhyun 
Fan, Bin

Fan, Hueng-Chuen

Fan, Wei

Fan, Zhichao

Faner, Rosa

Fang, Hsu-Wei

Fang, Ronnie

Fang, $\mathrm{Xi}$

Fang, Xiaolan

Fang, Yi

Fanizzi, Francesco P.

Fantacuzzi, Marialuigia

Fantuzzi, Laura

Faouzi, Malika

Fareed, Jawed

Faria, J.

Farnoud, Amir

Farr, Joshua

Farr, Tracy

Farrar, Mark

Farris, Stefano

Faruqi, Mohamed A.

Fasler-Kan, Elizaveta

Fast, Loren D.

Faundez, Victor

Faure, Sébastien

Fava, Paolo

Federico, Antonio

Fedorova, Olga V.

Fehrentz, Jean-Alain

Fei, Peiwen

Feinendegen, Ludwig

Felipe Farias, Davi

Felix, Klaus M.

Felix, Manuel

Felling, Ryan

Feng, Liu-Smith

Feng, Wenyi

Feng, Yibin

Feng, Yumei

Feng, Zhi Chao

Fenton, Robert

Feo, Francesco
Roh, Sangho

Rohira, Aarti

Rokstad, Anne Mari

Romagnoli, Stefano

Romani, Massimo

Romano, Andrea

Romano, Nicla

Romanov, Victor

Romee, Rizwan

Romei, Cristina

Romero Martínez, Ángel

Romero, Alejandro

Romero, Carmen

Romero-Sandoval, Alfonso

Ronca, Alfredo

Roncada, Paola

Ronchetti, Simona

Rong, Yueguang

Rooj, Arun

Roos, Jessica

Roper, Randall J.

Roque, Cláudio

Ros Lis, Jose Vicente

Ros, Marian A.

Rosales-Mayor, Edmundo

Rose, Giuseppina

Rosellini, Daniele

Rosendahl, Jonas

Ross, Ian

Ross, Ian L.

Rossa Jr., Carlos

Rossi, Simona

Rossignol, Julien

Rossman, Jeremy

Rossner, Pavel

Rostaing, Lionel

Rotllant, Josep

Rotomskis, Ricardas

Roubelakis-Angelakis, K.

Roubertoux, Pierre

Rouhiainen, Ari

Rouse, Matthew N.

Routledge, Michael 
Feraco, Alessandra

Fergani, Chrysanthi

Ferguson, David

Ferlini, Alessandra

Fernández, Ignacio

Fernández, Lucía

Fernández, Marta

Fernandez-Botran, Rafael

Fernandez-Jimenez, Nora

Fernández-Ponce, M. Teresa

Fernández-Rodríguez, Javier

Fernández-Ruiz, Javier

Ferrante, Claudio

Ferreira, Leonardo G.

Ferreira, Tracie L.

Ferreon, Josephine

Ferrera, Rene

Ferretti, Elisabetta

Ferrio, J. P.

Ferriol, Inmaculada

Fettkenhauer, Christian

Ficai, Anton

Fickert, Peter

Fiering, Steve N.

Figueiras, Edite

Figueiredo, Marxa L.

Figueroa, Carlos R.

Filek, Maria

Filella, Xavier

Filippova, Maria

Filleur, Stephanie

Filosa, Aldo

Filosto, Massimiliano

Fina, Emanuela

Findlay, David

Fine, James Burke

Finelli, Carmine

Fineschi, Vittorio

Fingerhut, Ralph

Finley, Brent

Finn, Aloke

Fioravanti, Antonella

Fiorucci, Stefano
Rouws, Luc

Roviello, Giandomenico

Roy, Deodutta

Roy, Jérôme

Roy, Kasturi

Royo, Felix

Roz, Luca

Rozanov, Dmitri

Rozhon, Wilfried

Rozot, Virginie

Rua, Diego

Ruan, Xiangbo

Rubino, Federico Maria

Rubio, Vicente

Rubis, Blazej

Rudd, Sean

Ruden, Douglas

Ruderman, Dan

Rudkin, Brian

Rudrabhatla, Sairam V.

Rudyk, Olena

Ruelland, Eric

Ruest, L. Bruno

Ruff, Adrian

Ruiter, Godard C. W. De

Ruiz, Sergio

Ruiz-Ederra, Javier

Ruiz-Gómez, Gloria

Ruiz-Lozano, Juan Manuel

Ruiz-Narváez, Edward A.

Rund, Deborah

Rupenthal, Ilva

Ruppert, J. Michael

Rupprecht, Rainer

Rurek, Michal

Russo, Giulia

Russo, José

Russo, Maria

Russo, Matteo A.

Russo, Pasquale

Russo, Suzanne

Ruzicka, Jiri

Ryffel, Bernhard 
Firestein, Bonnie L.

Fisch, Gene S.

Fisher, Aron B.

FitzHarris, Greg

Fixen, Kathryn

Fleckenstein, Johannes

Fletcher, Nicola F.

Flex, Andrea

Flieger, Jolanta

Fliniaux, Ophélie

Florczyk, Stephen J.

Florio, Tullio

Florsheim, Esther

Fonseca, Ana

Fonseca, Filomena

Fontalba-Navas, Andres

Fontana, Luca

Fontana, Robert J.

Fontes, Paulo

Foo, Eloise

Forcales, Sonia-V.

Ford, Henri

Formisano, Luigi

Formisano, Pietro

Forn-Cuni, Gabriel

Forneris, Federico

Forostyak, Serhiy

Forster, Britta

Forster, Jameson

Forster, John W

Fort, Patrice

Foster, John

Fosu-Nyarko, John

Fouassier, Laura

Foubert, Philippe

Fourtounas, Costas

Fox, Glen

Fozo, Elizabeth

Fracasso, Alessandra

Frachisse, Jean-Marie

Fradet-Turcotte, Amélie

Fraifeld, Vadim E.

France, Michael
Rylott, Elizabeth L

Ryu, Jong Hoon

Rzymowska, Jolanta

Saarma, Mart

Sacco, Angela

Sacco, Emilio

Saccone, Valentina

Sachmechi, Issac

Sachs, George

Sacitharan, Pradeep Kumar

Sadoghi, Patrick

Sadowska, Beata

Saelens, Xavier

Saera-Vila, Alfonso

Saez, Carmen

Safa, Ahmad

Sah, Hong-Kee

Saha, Achinto

Sahin, Mustafa

Saikumar, Pothana

Saini, Shikha

Sainlos, Matthieu

Saisho, Yoshifumi

Saito, Hideyuki

Saito, Yoshiro

Saiz-Fernández, Iñigo

Sajic, Marija

Sakaguchi, Yusuke

Sakai, Atsushi

Sakai, Masahiro

Sakai, Ryuichi

Sakamoto, Kei

Sakane, Fumio

Saki, Najmaldin

Sakuma, Tetsushi

Sala, Gianluca

Saladino, Raffaele

Salaspuro, Mikko

Salazar, Gloria

Salbach-Hirsch, Juliane

Salehin, Mohammad

Salek, Reza

Salgueiro, Carlos A. 


\begin{tabular}{|c|c|}
\hline Francisco González, Jorge & Salim, Vonny \\
\hline Franco, A. C. R. & Salio, Mariolina \\
\hline Franco, Diego & Saluk-Bijak, Joanna \\
\hline Franco, Luis & Salvador-Morales, Carolina \\
\hline Franco, Manuela Di & Salvarani, Nicolo' \\
\hline Franco, Rodrigo & Salvati, Bruno \\
\hline Francolini, Iolanda & Salvatore, Mirella \\
\hline Francolini, Maura & Salvi, Samanta \\
\hline Frangogiannis, Nikolaos & Samant, Rajeev S. \\
\hline Frank, Saša & Samarakoon, Rohan \\
\hline Frankel, Timothy L. & Sampathi, Shilpa \\
\hline Franken, Nicolaas & Samuel, Chrishan \\
\hline Franklin, Renty B. & Samuel, Marcus \\
\hline Franko, Andras & San Martin, Carmen \\
\hline Frantom, Patrick & Sanak, Marek \\
\hline Franzè, Annamaria & Sánchez, Beatriz Jurado \\
\hline Fraser, Justin F. & Sánchez-Pernaute, Olga \\
\hline Frau, Juan & Sanders, Alison P \\
\hline Freeman, Edward & Sands, Jeff M. \\
\hline Freeman, Jennifer L. & Sangiolo, Dario \\
\hline Freeman, Linnea R. & Sangiovanni, Enrico \\
\hline Frias, Juana & Sangireddy, Sasikiran Reddy \\
\hline Frieboes, Hermann & Sanij, Elaine \\
\hline Friedlander, Terence W. & Sanina, Nina \\
\hline Froemke, Robert C. & Sanjust, Enrico \\
\hline Frolov, Andrej & Sankaranarayanan, Nehru Viji \\
\hline Frolova, Liliya & Sanner, Michel F. \\
\hline Fromm, Bastian & Sanoh, Seigo \\
\hline Frotscher, Martin & Sansone, Clementina \\
\hline Frugis, Giovanna & Santamaría, Estrella \\
\hline $\mathrm{Fu}$, Junjie & Santamaria, Rita \\
\hline $\mathrm{Fu}$, Wen-Mei & Santambrogio, Paolo \\
\hline $\mathrm{Fu}$, Yongzhu & Santander, Javier \\
\hline Fuchs, Dietmar & Santander-Jiménez, Sergio \\
\hline Fujie, Tomoya & Santato, Clara \\
\hline Fujihara, Hisako & Santhakumar, Abishek \\
\hline Fujii, Tomomi & Santhosh, Sebastin M. \\
\hline Fujimori, Ko & Santin, Izortze \\
\hline Fujita, Mitsugu & Santino, Angelo \\
\hline Fujita, Tetsuji & Santolla, Maria Francesca \\
\hline Fujita, Yuichi & Santoni-Rugiu, Eric \\
\hline Fukuchi, Satoshi & Santoro, Cristina \\
\hline Fuller, Bryan & Santos, Maria Jacinta \\
\hline
\end{tabular}




\begin{tabular}{|c|c|}
\hline Fumagalli, Fabio & Santos-Gallego, Carlos \\
\hline Funaba, Masayuki & Santourlidis, Simeon \\
\hline Funder, John & Sanz, Ivan \\
\hline Funel, Niccola & Sanz-Clemente, Antonio \\
\hline Furfaro, Anna Lisa & Saper, Clifford \\
\hline Furness, John B. & Sarath, Gautam \\
\hline Fürnsinn, Clemens & Sarbini, Shahrul \\
\hline Furue, Masutaka & Sarkar, Sujan \\
\hline Furukawa, Toru & Sarker, Dipak \\
\hline Furuya, Hideki & Sarmast, Nima D. \\
\hline G., Musumeci & Sarniguet, Alain \\
\hline Gabbianelli, Rosita & Sarnowski, Tomasz \\
\hline Gaczynska, Maria & Sasaki, Masanori \\
\hline Gadaleta, Mariana & Sassi, Mohamed \\
\hline Gaddam, Ravinder Reddy & Sassi, Yassine \\
\hline Gaddameedhi, Shobhan & Satani, Nikunj \\
\hline Gadjeva, Mihaela & Satbhai, Santosh \\
\hline Gagos, Sarantis & Sather, Noah \\
\hline Gahete, Manuel D. & Satish, Latha \\
\hline Gahlaut, Vijay & Sato, Masa H. \\
\hline Gaida, Matthias & Sato, Takuichi \\
\hline Gajendrareddy, Praveen K. & Satoh, Shigeru \\
\hline Gajula, Rajendra & Satou, Ryousuke \\
\hline Gál, Peter & Satou, Tadaaki \\
\hline Galanis, Alex & Sattar, Sampurna \\
\hline Galat, Andrzej & Saturnino, Carmela \\
\hline Galeazzi, Roberta & Sau, Samaresh \\
\hline Galeffi, Francesca & Sauka, Diego \\
\hline Galek, Renata & Saunders, Nicholas \\
\hline Galetti, Maricla & Saurav, Kumar \\
\hline Galileo, Deni S. & Savaskan, Nicolai E. \\
\hline Galisteo Jr., Andres Jimenez & Saville, Robert \\
\hline Gall Troselj, Koraljka & Savion, Naphtali \\
\hline Gallardo, Rodrigo A & Savoie, Jean-Michel \\
\hline Galli, Dominique M. & Sawa, Teiji \\
\hline Gallicchio, Emilio & Sawada, Kenjiro \\
\hline Gallo, Linda & Sawai, Hirofumi \\
\hline Galuska, Sebastian & Scafoglio, Claudio \\
\hline Galve-Roperh, Ismael & Scahill, Lawrence \\
\hline Gambarotta, Giovanna & Scala, Valeria \\
\hline Gan, Boyi & Scandurra, Roberto \\
\hline Gan, Renyou & Scanes, Colin G. \\
\hline Gan, Ren-You & Schachter, Michael \\
\hline
\end{tabular}


Gangula, Pandu

Ganguly, Anutosh

Ganini, Douglas

Gantenbein, Benjamin

Ganz, Julia

Gao, Dian

Gao, Yanzhe

Gao, Yunxiang

García Hidalgo, Javier

Garcia Ivars, Jorge

Garcia, Lorena Carro

García-Escudero, Ramón

Garcia-Estañ, Luis Perez

Garcia-Gonzalez, Carlos A.

García-Martínez, Eva

García-Mauriño, Sofía

García-Villalón, Angel Luis

Gardin, Chiara

Gariboldi, Manuela

Garinis, George

Garrett, Scott

Garrido, Dolores

Garriga, Pere

Garrosa, Manuel

Gary-Bobo, Magali

Gasche, Christoph

Gaspar, Diana

Gaspar, Krisztian

Gáspár, Róbert

Gasparrini, Massimiliano

Gassler, Nikolaus

Gassman, Natalie R.

Gaszner, Balázs

Gatti, Laura

Gaudenzio, Nicolas

Gaudin, Robert

Gauger, Phillip C.

Gauthier, Patrick T.

Gavin, David P.

Gavira, José

Gavrilovic, Jelena

Gawarecka, Katarzyna

Gazerani, Parisa
Schaedler, Theresia A.

Schaefer, Jeremy

Schaefer, Michael

Schafer, Peter

Schäfer, Wilhelm

Schaffner, Walter

Schallenberg-Rüdinger, M.

Scharfman, Helen

Schefold, Joerg C.

Schembri, Mark

Schepens, Bert

Schepisi, Giuseppe

Scherberich, Arnaud

Scherer, Günther

Schernthaner, Gerit-Holger

Schettino, Giuseppe

Schiavone, Marco

Schiavone, Stefania

Schildgen, Oliver

Schiller, Dirk

Schinke, Thorsten

Schirmer, Bastian

Schisler, Jonathan

Schlaepfer, Isabel R.

Schlegel, Jürgen

Schlierf, Michael

Schlueter, Klaus-Dieter

Schmedake, Thomas A.

Schmid, Markus

Schmid, Michael

Schmid, Tobias

Schmidt, Anke

Schmidt, Martin

Schmidt, Mirko H. H.

Schmidt, Tannin

Schmidt-Arras, Dirk

Schmitt-Egenolf, Marcus

Schmutzhard, Erich

Schnapp, Lynn

Schneider, Achim G.

Schneider, Anja

Schneider, Barbara

Schob, Stefan 
Ge, Xiaodong

Gebel, Thomas

Gehrmann, Mathias

Geib, Scott

Geisler, Sarah

Gelmini, Stefania

Genetos, Damian

Geng, Feng

Gentile, Carla

Gentile, Piergiorgio

Georgakilas, Alexandros

George Parsons, K. S.

George, Sophia

George, Varghese

George, Vinoj

Georgia, Senta

Georgiev, Vasil

Georgieva, Radostina

Geraci, Fabiana

Gerardi, Carmela

Gerdol, Marco

Gericke, Martin

Gerke, Oke

Gerlach, Jared

Germain, Lucie

Germain-Aubrey, Charlotte

Geso, Moshi

Gewirtz, David A.

Ghaleh, Bijan

Ghayour-Mobarhan, Majid

Gherzi, Roberto

Ghosh, Biswarup

Ghosh, Sourav

Ghoshal, Kalpana

Giambona, Antonino

Giamperi, Laura

Giampieri, Francesca

Giannattasio, Sergio

Giannelli, Gianluigi

Giannoni, Patrizia

Giansanti, Francesco

Giaouris, Efstathios

Gibbison, Ben
Scholz, Sonja

Scholz-Starke, Joachim

Schomburg, Lutz

Schonthal, Axel

Schousboe, Arne

Schrag, Matthew

Schraufstatter, Ingrid U.

Schroeder, Dana

Schroit, Alan

Schubert, Mario

Schuhmann, Michael K.

Schulte, Jan Sebastian

Schultz, Liora

Schulz, Benjamin L.

Schulz, Richard

Schulz, Wolfgang A.

Schulze, Johannes

Schumacher, Anne

Schumacher, Julia

Schumann, Canan

Schütte, Kerstin

Schutte, Stacey C.

Schuur, Boelo

Schuurman, Henk-Jan

Schwartz-Albiez, Reinhard

Schwarz, Richard I.

Schwarz, Steven M.

Schweiger, Thomas

Schweiger, Wolfgang

Schweizer, Michael

Schwiebs, Anja

Scicchitano, Pietro

Scipione, Luigi

Scorziello, Antonella

Scott, Helton W.

Scotti, Marcus

Scotto D'Abusco, Anna

Scovassi, Anna Ivana

Scrima, Mario

Scudiero, Olga

Scudiero, Rosaria

Sebastian, Aimy

Seca, Ana 
Gijs, Marlies

Gilaberte, Yolanda

Gilch, Sabine

Gilding, Edward

Gilding, Edward K.

Giles, Walters

Gilli, Francesca

Gil-Mohapel, Joana M.

Gilson, Eric

Giordano, Antonio

Giosafatto, Valeria

Giovanni, Franzo

Giovarelli, Matteo

Girolomoni, Giampiero

Girouard, Hélène

Giuseppina, Andreotti

Giust, Davide

Głąbska, Dominika

Glading, Angela J.

Glasser, Wolfgang G.

Glick, Benjamin S.

Gloria, Antonio

Gnanamony, Manu

Gnanasekaran, Aswini

Gniewosz, Małgorzata

Gnocchi, Davide

Gobbi, Gabriella

Gobe, Glenda C

Gockel, Ines

Godfrey, Henry P.

Godschalk, Roger

Goedeke, Leigh

Gohda, Eiichi

Goicoechea, Nieves

Gok, Ozgul

Goldberg, Lynne J.

Goldhawk, Donna

Goldklang, Monica P.

Goldoni, Matteo

Goldring, Mary

Goldsmith, David R.

Goldstein, David

Golenhofen, Nikola
Seca, Ana M. L.

Sechi, GianPietro

Sediva, Anna

Sedmiková, Markéta

See, Violaine

Seeliger, Claudine

Seffens, William S.

Segall, Jeffrey E.

Segura, Ana

Sehrawat, Archana

Seifitokaldani, Ali

Seiler, Magdalene J.

Seimiya, Hiroyuki

Seiquer, Isabel

Seki, Naohiko

Seki, Yoshinobu

Sekine, Shuichi

Selby, Katja

Seliger, Corinna

Seligmann, Hervé

Selim, Sameh

Sella, Luca

Sellix, Michael T.

Selmin, Ornella I.

Selsby, Joshua

Semenov, Victor V.

Sempere, Lorenzo

Sengupta, Bidisha

Sengupta, Debrup

Senter, Peter D.

Seo, Cheong Hoon

Seo, Jungwon

Serafini, Gianluca

Sergaki, Maria Christina

Sergi, Consolato Maria

Serpico, Rosario

Serra, Dolors

Serra, Francisca

Servis, Michael

Sesaki, Hiromi

Seth, Devanshi

Sethi, Gautam

Setou, Mitsutoshi 
Goletti, Delia

Goltsov, Alexey

Gomes, Ana P.

Gomes, Maria Salomé

Gomes, Selma A.

Gómez De Cedrón, Marta

Gómez Llorente, Carolina

Gomez, Jordi

Gomez, Nidia

Gomez-Lopez, Nardhy

Gomez-Sanchez, Celso

Gomis-Rüth, F. Xavier

Gondhalekar, Ameya D.

Gondi, Christopher S.

Gondret, Florence

Gong, Fade

Gong, Limin

Gong, Siew-ging

Gonkowski, Slawomir

Gonzales, Cara B.

Gonzalez Bosc, Laura V.

Gonzalez, Antonio

González, Florenci V.

Gonzalez-Aguilar, Gustavo

Gonzalez-Barcala, F. J.

Gonzalez-conejero, Rocio

González-González, Rogelio

González-Maeso, Javier

González-Miguel, Javier

Gonzalez-Perez, Ruben R.

González-Vallinas, Margarita

Goppelt-Struebe, Margarete

Goreham, Renee

Gorgoulis, Vassilis

Goriely, Stanislas

Gorini Da Veiga, Ana

Gorman, Adrienne

Górnaś, Paweł

Gorodetsky, Raphael

Gorski, Jeffrey

Gortan Cappellari, Gianluca

Gosselet, Fabien

Goswami, Nandu
Setzer, William

Seva, Catherine

Sevcikova, Sabina

Seymour, Nikki

Sgarra, Riccardo

Sha, Zhe

Shabbir, Waheed

Shah, Dilip

Shah, Nilay

Shahbazi, Mohammad Ali

Shaikh, Mohamad Guftar

Shaikh, Mohd. Farooq

Shang, Li

Shankar, Eswar

Shanmugam, Muruganandan

Shannon, Oonagh

Shao, Fangwei

Shao, Kan

Shapiro, Allison L. B.

Sharma, Alok

Sharma, Anjali

Sharma, Anurag L.

Sharma, Atul

Sharma, Bhesh

Sharma, Himanshu

Sharma, Naveen

Sharma, Rohit

Sharma, Sudha

Sharma, Sushil

Shaw, Lisa

Shaw, Odette

Shecterle, Linda M.

Sheen, Shyn-Shin

Sheikh, Nadeem

Shelton, Holly

Shemanko, Carrie

Shemfe, Mobolaji

Shen, Che-Kun James

Shen, Szu-Chuan

Shen, Yang

Shen, Zhenyu

Sheppard, Mary N.

Sherbenou, Daniel 
Gotea, Valer

Goua, Marie

Goukassian, David

Gouveia, Susana

Gowans, Eric

Goyal, Rajni

Gozzini, Antonella

Grabrucker, Andreas M.

Gradoni, Luigi

Graf, Gregory

Graham, Regina

Graier, Wolfgang

Gräler, Markus

Gralinski, Lisa

Gramlich, Michael

Grammatikakis, Ioannis

Gramzow, Lydia

Granados-Principal, Sergio

Grande, Fedora

Granja, Aitor

Granot, David

Grant, George

Grant, William

Grãos, Mário

Grapputo, Alessandro

Grases, Felix

Grassi, Gabriele

Gray, Clint

Graziani, Maristella

Graziano, Guella

Grazul-Bilska, Anna

Grazzi, Licia

Grbeša, Darko

Greene, Catherine M.

Greene, Lesley H.

Greene, Paul E.

Greenfield, John

Greenhalgh, David

Greenwood, Michael T.

Gregory, Richard L.

Greither, Thomas

Grether-Beck, Susanne

Grewal, Raji Paul
Sherrard, Rachel

Shertzer, Howard G.

Shewale, Swapnil

Shi, Hua

Shi, Jiaqi

Shi, Yunhua

Shiao, Young-Ji

Shigdar, Sarah

Shih, Yu-Ru

Shiina, Takashi

Shim, Yhong-hee

Shimada, Tsutomu

Shimada, Yasuhito

Shimura, Hanako

Shinoda, Kei

Shiozawa, Yusuke

Shipman, Kate

Shirahata, Tatsuya

Shiraishi, Takehiko

Shiue, Yow-ling

Shokal, Upasana

Sholkamy, Eman

Shostak, Anton

Shpak, Elena D.

Shteinberg, Michal

Shukla, Alpana P.

Shukla, Girish

Shukla, Sanjeev

Shulman, Adrian

Shysh, Angela M.

Sibilla, Sara

Sibille, Etienne L.

Sidari, Rossana

Sidiropoulos, Christos

Sidorenko, Viktoriya S.

Sié, Pierre

Sieber, Fritz

Siebuhr, A. S.

Sier, Cornelis

Sierecki, Emma

Sikkema-Raddatz, Birgit

Silina, Yuliya

Silipo, Alba 
Grewe, Felix

Grieco, Domenico

Grieco, Francesco

Grillari, Johannes

Grimholt, Unni

Grimm, Marcus

Grinberg, Daniel

Grinfeld, Jacob

Grishin, Nick

Groh, Isabel Anna Maria

Gromadzka, Karolina

Grones, Peter

Grootaert, Charlotte

Gros, Frédéric

Grösch, Sabine

Gross, Christina

Gross, Joshua B.

Grosse, Jirka

Grossi, Giancarlo

Grossmann, Tom N.

Grothusen, Christina

Grotta, James C.

Groveman, Bradley

Gruber, Tanja

Grumet, Rebecca

Grzebelus, Dariusz

Grzesiuk, Elżbieta

$\mathrm{Gu}$, Feng

$\mathrm{Gu}$, Liuqi

Gu, Zhen

Guan, Bin

Guardado-Calvo, Pablo

Gubin, Denis

Guedens, Wanda

Guéguen, Laurent

Guerini, Franca R.

Guerlesquin, Francoise

Guerriero, Gea

Gugatschka, Markus

Guha, Prajna P.

Guha, Sonia

Guhathakurta, Subhrangshu

Guidotti, Alessandro
Sill, Heinz

Silva, Gabriela Jorge

Silva, Luís R.

Silva, Margarida F. B.

Silva, Nuno A.

Silva, Susana N.

Silva-Fisher, Jessica

Silvestri, Giuliana

Silvestri, Ida

Silvestri, Romano

Silvestris, Nicola

Simes, Dina

Simko, Fedor

Simkó, Myrtill

Simmons, Peter A.

Simões, Marta Filipa

Simon, Marie-Christine

Simon, Sanford M.

Simon, Scott I.

Simoni, Jan

Simonsson, Stina

Simpson, Craig

Simpson, Julie E.

Singer, Esther

Singh, Amareshwar

Singh, Anil

Singh, Atul

Singh, Atul Kumar

Singh, Bhagirath

Singh, Chandra K.

Singh, Chanpreet

Singh, Guramrit

Singh, Jugpreet

Singh, Nisha

Singh, Rajbir

Singh, Rajesh

Singh, Sonal

Singh, Vijay K.

Singh, Vishal

Sinha, Amit

Sinha, Kalyan

Siniscalco, Dario

Sinn, Marianne 
Guignabert, Christophe

Guil, Sònia

Guilpain, Philippe

Guimaraes, Fernando

Gulati, Pankaj

Gulick, Patrick J.

Gundara, Justin

Gunia-Krzyżak, Agnieszka

Guo, Fenghai

GUO, PU

Guo, Tai L.

Guo, Weimin

Guo, Zong Sheng

Gupta, Ankit

Gupta, Sudhiranjan

Gupta, Vivek K.

Gurha, Priyatansh

Gurikov, Pavel

Gursoy, Gamze

Gustafsson, Rasmus

Gutiérrez, Raúl Sánchez

Gutiérrez-Juárez, Roger

Guzmán, Esther A.

Gwinn, William M.

H Van Der Horst, Edward

Ha, Sandie

Haase, Hajo

Habiba, Khaled

Haboubi, Nadim

Hachiya, Tsuyoshi

Hack, Ethan

Hackenberg, Stephan

Hackl, Matthias

Hadjiargyrou, Michael

Hadjipavlou-Litina, Dimitra

Hadler-Olsen, Elin

Haefliger, Simon

Haenen, Guido

Hagenlocher, Yvonne

Hagerman, Randi J.

Hagmann, Sébastien

Hait, Nitai

Hallberg, Bengt
Siomi, Harukiko

Sipponen, Mika Henrikki

Sirdah, Mahmoud

Sirotkin, Alexander V.

Sisley, Stephanie

Sissung, Tristan M.

Sista, Subhash

Sita, Giulia

Sitaru, Cassian

Si-Tayeb, Karim

Sivakumar, Sushama

Sivasubramaniyan, Kavitha

Siwek, Agata

Sizochenko, Natalia

Skalko-Basnet, Natasa

Skalova, Lenka

Skendros, Panagiotis

Skibicka, Karolina

Skov, Jeppe

Skov, Per Stahl

Skundric, Dusanka S.

Slama-Schwok, Anny

Slattery, David

Slavik, Roger

Sleutels, Tom

Slieker, Roderick C.

Sloan, Kenneth B.

Słoczyńska, Karolina

Slominski, Andrzej

Slot, Jason C.

Slouka, Christoph

Slovin, Susan

Słupecka, Monika

Smagghe, Guy

Smani, Younes

Smarr, Benjamin L.

Smart, Neil

Smartt, Chelsea T.

Smed-Sörensen, Anna

Smeets, Eric E.

Smekalova, Elena M.

Smeriglio, Antonella

Śmieszek, Agnieszka 


\begin{tabular}{|c|c|}
\hline Hall-Mendelin, Sonja & Smirnovas, Vytautas \\
\hline Hamada, Fumika & Smith, Brittany L. \\
\hline Hamaguchi, Masahide & Smith, Ellen M. Lavoie \\
\hline Hamanishi, Junzo & Smith, Ernest E. \\
\hline Hamann, Martine & Smith, G. M. \\
\hline Hammer, Suntrea & Smith, Jessica \\
\hline Hammers, Christoph M. & Smith, Mark \\
\hline Hammerschlag, Margaret R. & Smith, Micholas Dean \\
\hline Hampel, Daniela & Smith, Stephen M. \\
\hline Han, Dong Wook & Smith, Tim A. D. \\
\hline Han, Jaehong & Smutzer, Gregory \\
\hline Hanai, Jun-ichi & Smýkal, Petr \\
\hline Hanaoka, Mitsumasa & Smyth, Lesley A. \\
\hline Hancock, Robert & Sneddon, Jennifer \\
\hline Hane, James & Sneyd, Mary Jane \\
\hline Hanke, Tomáš & Soares, Claudio M. \\
\hline Hannibal, Luciana & Soenen, Stefaan J. \\
\hline HANO, Christophe & Sohl, Christal \\
\hline Hanover, D John A. & Sohn, Young Chang \\
\hline Hans, Guy & Sokolova, Viktoriya \\
\hline Hanschen, Franziska S. & Solà Alberich, Rosa \\
\hline Hanson, Peter & Solano, Francisco \\
\hline Hanss, Michel & Soldevilla, Mario \\
\hline Hanus-Fajerska, Ewa & Soleri, Daniela \\
\hline Haque, Inamul & Soler-Vasco, Laura \\
\hline Haque, Rashidul & Solomon, Matia \\
\hline Hara, Akira & Solorio, Luis \\
\hline Harada, Mamoru & Son, Deok-soo \\
\hline Harada, Shuko & Son, Mi-Won \\
\hline Harashima, Nanae & Song, Chunhua \\
\hline Hardeland, Rudiger & Song, Jeongmin \\
\hline Hardingham, Jennifer E. & Song, Jinhua \\
\hline Hardy, John G. & Song, Minseok \\
\hline Hariharan, Seethalakshmi & Song, Su \\
\hline Haro, Diego & Sonkoly, Enikö \\
\hline Harper, Alan & Sonnier, Rodolphe \\
\hline Harris, Randall E. & Sonntag, Kai-christian \\
\hline Harris, William & Sonsalla, Patricia K. \\
\hline Harrison, Jonathan S. & Soole, Kathleen \\
\hline Harrison, Mark & Soopramanien, Anba \\
\hline Har-Shai, Yaron & Sorriento, Daniela \\
\hline Hart, Martin & Sosa, Silvio \\
\hline Hartshorn, Kevan & Sosna, Justyna \\
\hline
\end{tabular}




\begin{tabular}{|c|c|}
\hline Harvey, Adam & Sosne, Gabriel \\
\hline Harvey, Ruth & Sotillo, Javier \\
\hline Has, Cristina & Soudeyns, Hugo \\
\hline Hasanuzzaman, Mirza & Soulika, Athena \\
\hline Hasegawa, Midori & Sounni, Nor Eddine \\
\hline Hasegawa, Morifumi & Sousa, Célia T. \\
\hline Hasenbein, Simone & Sousounis, Konstantinos \\
\hline Hashimoto, Makoto & Souza, Glauco R. \\
\hline Hashimoto, Naoto & Souza, Rhonda Frances \\
\hline Hassan, Iman & Soveral, Graça \\
\hline Hassan, Mohamed & Soverini, Simona \\
\hline Hassan, Sherif T. S. & Spanakis, Marios \\
\hline Hassouna, Rim & Spandou, Evangelia \\
\hline Hata, Aaron & Spano, Giuseppe \\
\hline Hatano, Tsutomu & Sparacino-Watkins, Courtney \\
\hline Hatfield, Ronald D. & Spassov, Sashko \\
\hline Hatfield, Stephen M. & Spelman, Lynda \\
\hline Hattori, Yuichi & Spengler Neff, Anet \\
\hline Hatzis, Pantelis & Spengler, Dietmar \\
\hline Haubner, Frank & Sperling, Mark A. \\
\hline Hauser, Michael Arthur & Spetz, Johan \\
\hline Hausman, G. J. & Spickett, Corinne \\
\hline Havemeyer, Antje & Spiegel, Martin \\
\hline Haverkamp, Richard G. & Spiewak, Radoslaw \\
\hline Haworth, Oliver & Spitzner, Melanie \\
\hline Hayakawa, Tohru & Spoerl, David \\
\hline Hayakawa, Yoshihiro & Spring, Kevin \\
\hline Hayashida, Morihiro & Spyridopoulos, Ioakim \\
\hline Hayer-Hartl, Manajit & Sreedasyam, Avinash \\
\hline Hayes, Alan & Sreedhar, Remya \\
\hline Hayes, Sidney & Sreerama, Subramanya \\
\hline Haynes, David & Sridhar, Jayalakshmi \\
\hline Haynes, Erin & Srihari, Sriganesh \\
\hline Haynes, Kathleen G & Srivastava, Akhil \\
\hline Hazak, Ora & Srivatsan, Eri S. \\
\hline Hazane-Puch, Florence & Srivatsan, Malathi \\
\hline $\mathrm{He}$, Fei & Srivenugopal, Kalkunte \\
\hline He, Hongliang & St John, James A. \\
\hline He, Kangmin & Stacchiotti, Alessandra \\
\hline He, Peijian & Stacey, M. \\
\hline Heaney, J. L. & Stadler, Marc \\
\hline Heaphy, Christopher & Stafford, Philip \\
\hline Hearn, Michael J. & Stagos, Dimitrios \\
\hline
\end{tabular}


Hebbard, Lionel

Heber, David

Heberden, Christine

Hedges, Jodi

Heery, David M.

Hefferon, Kathleen

Hegedüsová, Alžbeta

Hegyi, Eszter

Hehlgans, Stephanie

Heidari, Mohammad

Heidorn-Czarna, Malgorzata

Heiker, John T.

Heimburg-Molinaro, Jamie

Helfield, Brandon L.

Heller, Gerwin

Hellewell, Sarah

Hellstrom, Karl Erik

Hellweg, Christine E.

Hellwig, Michael

Henderson, W. Matthew

Hennequin, Claire

Henry, Michael E.

Heo, Soo-Jin

Hepworth, Chris

Her, Lu-Shiun

Heras, Joseph

Hermann, Marcela

Hermanns, Heike

Hernández-Hernández, A.

Hernández-Rodríguez, César

Herndon, David

Herr, Deron Raymond

Herranz, Beatriz

Herrero, Baudilio

Herrero, María Jesús

Herrero-Beaumont, Gabriel

Hershfinkel, Michal

Hertz, Daniel L.

Hess, Angela

Hesson, Luke

Hettinga, Kasper

Hewitt, Kevin Cecil

Hickey, Michael J.
Ståhle, Mona

Staines, Katherine Ann

Stallcup, William

Stambuk, Nikola

Stančiaková, Lucia

Stancu, Andreea Lucia

Standage, Daniel S.

Staneva, Desislava

Stanford, John

Staniszewska, Monika

Stanley, Jone

Stanton, Robert C.

Starostik, Petr

Staunstrup, Nicklas Heine

Stavrianidi, Andrey

Stecker, Mark M.

Steel, Laura F.

Stefanidou, Maria E.

Stefanini, Lucia

Stefano, Caldaralli

Stefano, Cassanelli

Steinmetz, Michel O.

Stenbeck, Gudrun

Stephan, Holger

Stępień, Łukasz

Stevanato, Piergiorgio

Steven, Sebastian

Stevenson, Bradley

Stewart, Cameron R.

Stewart, James

Stewart, Jason

Stewart, Lamonica V.

Steyn, Frederik

Stillaert, Filip

Stilli, Donatella

Stillman, Martin

Stingeni, Luca

Stiuso, Paola

Stivrins, Normunds

Stobdan, Tsering

Stobiecki, Maciej

Stocco, Carlos

Stocker, Reto 
Higashi, Tomomi

Higuchi, Kazuhide

Hii, Charles S. T.

Hildreth, Blake

Hildt, Eberhard

Hileman, Lena

Hill, Hellen Z.

Hillard, Cecilia

Himeno, Hyouta

Hinds Jr., Terry D.

Hininger-Favier, Isabelle

Hinkelbein, Jochen

Hinojosa, Silvia

Hinz, Sebastian

Hirabayashi, Jun

Hiraga, Toru

Hirakawa, Hidehiko

Hirano, Katsuya

Hirano, Tomoko

Hirano, Tomoya

Hirasawa, Noriyasu

Hirasawa, Takashi

Hirbe, Angela C.

Hirsch, Pierre

Hitchins, Megan

Hitomi, Hirofumi

Hla, Timothy

Ho, Chi-Tang

Ho, Jar-Yi

Ho, Mengfei

Ho, Roger Chun-Man

Ho, Shin-Lon

Ho, Wen-Fu

Ho, Wing Shing

Hoang-Vu, Cuong

Hochberg, Irit

Höckner, Martina

Hodgkinson, James

Hoemann, Caroline

Hoff, Rodney

Hoffmann, Klaus

Hoffmann-Benning, Susanne

Hofmann, Johann
Stokes, Karen Y.

Stone, Kari

Stone, Trevor William

Stonehouse, Nicola

Stopeck, Alison T.

Stopka, Pavel

Stopka, Tomáš

Štorchová, Helena

Storer, Nicholas P.

Stork, Christian J.

Stout, David A.

Stowasser, Michael

Stowell, Kathryn M.

St-Pierre, Yves

Stratmann, Bernd

Strauss, Sarah

Strillacci, Maria Giuseppina

Strohbach, Anne

Stroka, Deborah M.

Strong, Cristina De Guzman

Strudwick, Xanthe

Strzemiecka, Beata

Studzinski, George P.

Stumpel, Connie T. R. M.

Stunes, Astrid Kamilla

$\mathrm{Su}$, Jianguo

Su, Rui

$\mathrm{Su}$, Ting

Suarez-Cunqueiro, M. M.

Suau, Pedro

Subramaniam, Prasad

Sudhir, Putty-Reddy

Sugahara, Takuya

Sugawara, Akira

Sugimoto, Naotoshi

Sugimoto, Sachiko

Sugimoto, Yukio

Sugimura, Haruhiko

Sugita, Noriko

Sugiura, Daisuke

Sukhov, Vladimir

Sukocheva, Olga

Sun, Fengjie 
Hogstrand, Christer

Höhfeld, Jörg

Hojjat-Farsangi, Mohammad

Holalu, Srinidhi

Holder, Alvin A.

Holen, Ingunn

Holland, Olivia

Höllig, Anke

Hollomon, Mario G

Hollywood, Mark

Holmes, Dawn E.

Holmøy, Trygve

Holst, Otto

Holt, Jeffrey

Holt, Scott M.

Holzmann, Klaus

Homa, Joanna

Homma, Miwako Kato

Hon, Kam-Lun

Hondermarck, Hubert

Hong, Yonggeun

Hood, Sean

Hooijberg, Erik

Hooper, Cornelia

Hopperton, Kathryn

Hori, Yuichiro

Hori, Yuuichi

Horohov, David W.

Horrocks, A. Richard

Horvath, David P.

Horvath, György

Hosick, Peter

Hoskins, Clare

Hosmane, Narayan

Hosohata, Keiko

Hosur, Vishnu

Hou, Anfu

Hou, Chien-Wei

Houen, Gunnar

Houshmand, Sina

Houston, Jessica

Houston, Mark C.

Hove-Jensen, Bjarne
Sun, Jie

Sun, Liang

Sun, Nawei

Sun, Qian

Sun, Yingjie

Sun, Yuan

Sun, Yuxiang

Sundar, Isaac Kirubakaran

Sundar, Reshma

Sunseri, Francesco

Suntharalingam, K.

Surapathrudu, Kanakala

Surdacki, Andrzej

Surguchov, Andrei

Sussman, Michael R.

Sutaria, Dhruvitkumar

Sutherland, John B.

Suzuki, Hideo

Suzuki, Jon Y.

Suzuki, Nobuhiro

Suzuki, Takehiro

Suzuki, Takuya

Svenningsen, Åsa Fex

Svensson, Katrin J.

Swamy, Ganesh

Swarup, Vimal

Sweazea, Karen

Sweetman, Martin

Swevers, Luc

Świeca, Michał

Swindle-Reilly, Katelyn E.

Syed, Nelofer

Syed, Viqar

Sykes, Erin K.

Sykora, Peter

Sylwester, Slusarczyk

Sytykiewicz, Hubert

Szablewski, Leszek

Szabo, Aron

Szarmach, Arkadiusz

Szebeni, Gabor Janos

Széll, Márta

Szewczyk, Bernadeta 


\begin{tabular}{|c|c|}
\hline Hoving, Hilde & Szewczyk, Katarzyna \\
\hline Howarth, Gordon & Szilagyi, Andras \\
\hline Howell, Viive & Szklarczyk, Damian \\
\hline Hoying, James B. & Szmidt, Alfred E. \\
\hline Hrabal, Richard & Szopa, Agnieszka \\
\hline Hribal, Marta & Szostak, Michael P. \\
\hline Hrobárik, Peter & Szumny, Antoni \\
\hline Hrstka, Roman & Szyszkowicz, Mieczyslaw \\
\hline Hsia, Shih-Min & T. M. De Rosales, Rafael \\
\hline Hsiao, George & Tababat-Khani, Poya \\
\hline Hsieh, Hsi-Lung & Tabata, Yasuhiko \\
\hline Hsieh, James J. & Tabrizi, Mojgan Aghazadeh \\
\hline Hsieh, Tusty-Jiuan & Tacconi, Carlotta \\
\hline Hsieh, Yi-Hsien & Tachibana, Yuya \\
\hline Hsin, Kun-Yi & Tadić, Vanja \\
\hline Hsu, Erin L. & Taghibiglou, Changiz \\
\hline Hsu, Kuo-Sheng & Taguchi, Ayumu \\
\hline Hsu, Shu-hao & Taguchi, Y. H. \\
\hline Hsu, Todd & Tahimic, Candice \\
\hline Hsu, Ya-Ling & Taiho, Kambe \\
\hline Hsueh, Yi-Huang & Taiki, Aoshi \\
\hline $\mathrm{Hu}$, Chao & Tajima, Shoji \\
\hline Hu, Michael Sung-Min & Tajima, Soichiro \\
\hline $\mathrm{Hu}, \mathrm{Min}$ & Takabe, Kazuaki \\
\hline $\mathrm{Hu}$, Ming-Chang & Takac, Tomas \\
\hline Hu, Wei-Gang & Takagi, Kiyoshi \\
\hline $\mathrm{Hu}$, Yang & Takahashi, Daisuke \\
\hline Huang, Chiu-jung & Takahashi, Hiroki \\
\hline Huang, Guan-Jhong & Takahashi, Ryou-u \\
\hline Huang, Нao & Takahisa, Kanekiyo \\
\hline Huang, Jason C. & Takaki, Akinobu \\
\hline Huang, Jian & Takaki, Manabu \\
\hline Huang, Lei & Takano, Katsura \\
\hline Huang, Meng-Yuan & Takano, Toshiyuki \\
\hline Huang, Nai-Kuei & Takata, Minoru \\
\hline Huang, Shaobai & Takatsuji, Hiroshi \\
\hline Huang, Shyh-Shyun & Takatsuka, Jun \\
\hline HUANG, Wen-Chin & Takayama, Kei \\
\hline Huang, Wen-Lii & Takayama, Kenichi \\
\hline Huang, Wenlin & Takayama, Yoshiharu \\
\hline Huang, Xingfeng & Takegahara, Noriko \\
\hline Huang, Yan-Jang & Takeoka, Shinji \\
\hline Huang, Ying-Hsien & Takumi, Shigeo \\
\hline
\end{tabular}


Huang, Yu-Tzu

Huber, Samuel

Huber, Veronica

Huettmann, Falk

Hughson, Michael D.

Hulot, Jean-Sébastien

Hulse-Kemp, Amanda

Hung, Jan-jong

Hunt, Piper Reid

Huppert, Volker

Huppi, Konrad

Huppler, Anna R.

Hura, Tomasz

Husnjak, Koraljka

Hutchinson, Edward

Hutmacher, Dietmar

Hüttenberger, Dirk

Huwiler, Andrea

Hwang, In Koo

Hwang, Ki-Chul

Hwang, Yong-sic

Hwang, Yongsung

Hymowitz, Sarah

Hyun, Kyung-Yae

Hyun, Sang-Hwan

Iaccino, Enrico

Iacobellis, Gianluca

Iacono, Pierluigi

Iadarola, Paolo

Ibeanu, Gordon

Ibiza, Sales

Ichikawa, Hiroshi

Ichimura, Kazuya

Ieraci, Alessandro

Iglesias-Fernández, Raquel

Ijima, Hiroyuki

Ijiri, Daichi

Ikeda, Masahiro

Ikegaya, Naoki

Iles, Ray

Ilies, Marc A.

Iliopoulos, Konstantinos

Illuminati, Giulio
Tallerico, Rossana

Talò, Giuseppe

Talukder, Shyamal Krisna

Tamama, Kenichi

Tamara, Varcoe

Tamás, Ladislav

Tamayol, Ali

Tamma, Grazia

Tan, Bee K.

Tan, Dun-Xian

Tan, Gene S.

Tan, Sih Min

Tan, Xiangshi

Tanabe, Shihori

Tanaka, Ayumi

Tanaka, Hiromitsu

Tanaka, Hiroyuki

Tanaka, Nobuyuki

Tanaka, Takashi

Tanaka, Takuji

Tanaka, Tamotsu

Tanaka, Tomoaki

Tanaka, Toshiaki

Tang, Ming-Jer

Tang, Yu

Tanoi, Keitaro

Tanonaka, Kouichi

Tan-Wilson, Anna L.

Tao, Shasha

Tao-Hsin, Tung

Tappia, Paramjit S.

Tarafder, Solaiman

Tarasova, Nadya I.

Tarn, Woan-yuh

Tarr, D. Ellen

Tarro, Giulio

Taruno, Akiyuki

Tashima, Toshihiko

Tashiro, Hirotaka

Tata, Ada Maria

Tate, Rothwelle J.

Tateishi, Kensuke

Tatsuke, Tsuneyuki 


\begin{tabular}{|c|c|}
\hline Ilmer, Matthias & Tatullo, Marco \\
\hline Im, Seung-Soon & Taube, Joe \\
\hline Im, Sin-hyeog & Taubert, Andreas \\
\hline Imai, Kazushi & Taura, Kojiro \\
\hline Imaizumi, Takato & Tausk, Francisco \\
\hline Imlach, Wendy L. & Tavares, Anthony \\
\hline Inaba, Hiroshi & Tavazzi, Barbara \\
\hline Inagaki, Noritoshi & Tawil, Bill J. \\
\hline Inamura, Kentaro & Taylor, Caroline \\
\hline Incitti, Tania & Taylor, Elaine \\
\hline Indiveri, Cesare & Taylor, Graham \\
\hline Ingels, Helene & Taylor, Juliet M. \\
\hline Ingley, Evan & Taylor, Kathryn \\
\hline Ingram-Smith, Cheryl & Taylor, Matthew \\
\hline In-Jung, Kim & Taylor, T. Matthew \\
\hline Inman, Sharon & Tazawa, Hiroshi \\
\hline Inoue, Atsuko & Te Riele, Hein \\
\hline Inselman, Amy & Te Velthuis, Aartjan \\
\hline Intaglietta, Marcos & Tedbury, Philip \\
\hline Inui, Hideyuki & Tedesco, Serena \\
\hline Inuzuka, Hiroyuki & Tee, Andrew R. \\
\hline Ioanna, Andreadou & Teeri, Teemu \\
\hline Iordanskiy, Sergey & Teets, Nicholas \\
\hline Iorio, Egidio & Teige, Markus \\
\hline Iorio, Marilena & Teixeira, Renake N. \\
\hline Iorio, Ronald M. & Teixidó, Elisabet \\
\hline Iorizzo, Massimo & Tejedo, Juan R \\
\hline Iqbal, M. Anwar & Tejero, Jesús \\
\hline Iqbal, Tariq H. & Tellone, Ester \\
\hline Iraci, Nunzio & Telysheva, Galina \\
\hline Irato, Paola & Templeton, Dennis J. \\
\hline Irimia, Daniel & Tencerova, Michaela \\
\hline Irimura, Tatsuro & Teng, Ru-Jeng \\
\hline Irvine, Katharine M. & Teng, Shaolei \\
\hline Isaji, Shuji & Tenta, Roxane \\
\hline Isani, Gloria & Tentolouris, Nicholas \\
\hline Isemura, Mamoru & Teodoro, Jose G. \\
\hline Isenberg, Jeffrey S & Teoh, Eugene \\
\hline Ishibashi, Kenichi & Teotia, Pooja \\
\hline Ishihara, Hisamitsu & Ter Beek, Josy \\
\hline Ishihara, Kengo & Terajima, Masanori \\
\hline Ishii, Isao & Terracciano, Daniela \\
\hline Ishii, Kazunari & Terrier, Olivier \\
\hline
\end{tabular}




\begin{tabular}{|c|c|}
\hline Ishii, Tetsuro & Terry, Randall G. \\
\hline Ishikawa, Kiyotake & Teruel-Montoya, Raúl \\
\hline Ishikawa, San-e & Terzaghi, William \\
\hline Ishimoto, Takatsugu & Teschke, Rolf \\
\hline Ishita, Chatterjee & Testai, Fernando D. \\
\hline Ishiyama, Katsuya & Teusch, Nicole \\
\hline Ishizaki, Takuma & Tevosian, Sergei G. \\
\hline Islam, M. Nurul & Tezil, Tugsan \\
\hline Islam, Md Soriful & Thakkar, Prashant \\
\hline Isshiki, Yoshiaki & Thakur, Chitra \\
\hline Ita, Kevin & Thannhauser, Theodore W \\
\hline Ito, Ken-ichi & Theile, Dirk \\
\hline Ito, Kousei & Theiss, Arianne L. \\
\hline Ito, Shosuke & Thelin, Eric \\
\hline Itoh, Masanori T. & Thi, Mia Mia \\
\hline Itoh, Yoshifumi & Thiel, Gerald \\
\hline Itou, Junji & Thiel, Volker \\
\hline Ittner, Arne & Thiem, Kathrin \\
\hline Ivancic Santek, Mirela & Thierry, Hauet \\
\hline Ivanov, Alexander V & Thijs, Sofie \\
\hline Ivanova, Lyudmila & Thilmony, Roger \\
\hline Ivask, Angela & Thiriet, Marc \\
\hline Iwaizumi, Masakazu G. & Thiruvengadam, Muthu \\
\hline Iwamori, Masao & Thomas, Seddon Y. \\
\hline Iwamoto, Sadahiko & Thomas, T. John \\
\hline Izawa, Takashi & Thomas, Wayne \\
\hline Izawa, Takeshi & Thompson, Crista \\
\hline Izquierdo-Useros, Nuria & Thompson, Loren P. \\
\hline Izsvák, Zsuzsanna & Thompson, Van Purdy \\
\hline Izumiya, Yoshihiro & Thoms, Kai-Martin \\
\hline Jablonska, Ewa & Thorpe, Chavaunne \\
\hline Jackson, Christopher & Thounaojam, Menaka \\
\hline Jackson, Daniel & Threadgill, Michael \\
\hline Jacob, Francis & Threapleton, Diane Erin \\
\hline Jacobs, Aaron T. & Thrivikraman, Greeshma \\
\hline Jacobs, Enno & Thunström, Erik \\
\hline Jacobs, Miriam & Tian, Bin \\
\hline Jacobson, Lauren & Tiemann-Boege, Irene \\
\hline Jacova, Claudia & Tiffen, Jessamy C. \\
\hline Jacquet, Alain & Tiidus, Peter \\
\hline Jadavji, Nafisa M. & Tille, Jean-Christophe \\
\hline Jaffar, Zeina & Tilley, Michael \\
\hline Jagiello, Karolina & Timme-Laragy, Alicia R. \\
\hline
\end{tabular}




\begin{tabular}{|c|c|}
\hline Jahandideh, Samad & Timoshenko, Alexander V. \\
\hline Jain, Gaurav & Timperio, Anna Maria \\
\hline Jain, Rachit & Tinuper, Paolo \\
\hline Jaing, Tang Her & Tiso, Natascia \\
\hline Jaiswal, Amit K. & Titorenko, Vladimir I. \\
\hline Jaiswal, Ashvin R. & Tiwari, Sanjay \\
\hline Jaiswal, J. K. & To, Kin-Ying \\
\hline Jakobi, Tobias & Tobimatsu, Yuki \\
\hline Jakovcevski, Mira & Tobita, Hiroyuki \\
\hline Jakubikova, Jana & Toden, Shusuke \\
\hline Jakubzick, Claudia V. & Todorovic, Slobodan \\
\hline Jaligot, Estelle & Togbe, Dieudonnée \\
\hline James, Euan & Toh, Wei Seong \\
\hline Janakiraman, Harinarayanan & Toldra, Fidel \\
\hline Janda, Tibor & Toledo-Ortiz, Gabriela \\
\hline Janecka, Anna & Tolkach, Yuri \\
\hline Jang, An-Soo & Tolosa, Ezequiel \\
\hline Jang, Seonghoe & Tomar, Dhanendra \\
\hline Janga, Madhusudhana & Tomasetti, Carmine \\
\hline Jantschitsch, Christian & Tomaz, Cândida Teixeira \\
\hline Januchowski, Radosław & Tombesi, Sergio \\
\hline Janusz, Maria & Tomita, Hiroyuki \\
\hline Jaradat, Nidal & Tomitaka, Asahi \\
\hline Jara-Palacios, M. José & Tommasini, Steven M. \\
\hline Jareño-Esteban, José Javier & Tomuta, Ioan \\
\hline Jarząb, Barbara & Toniolo, Antonio Q. \\
\hline Jat, Parmjit S. & Töreyin, Hakan \\
\hline Jaumot, Joaquim & Torras, Joan \\
\hline Jawien, Jacek & Torres Lagares, Daniel \\
\hline Jayant, Rahul Dev & Torres López, Ma Isabel \\
\hline Jayaram, Lata & Torres Perales, Carolina \\
\hline Jazirehi, Ali & Torres-Aleman, Ignacio \\
\hline Jellyman, J. K. & Torres-Benito, Laura \\
\hline Jeltsch, Albert & Torres-Reveron, Annelyn \\
\hline Jembrek, Maja Jazvinšćak & Torzewski, Michael \\
\hline Jena, Bhanu P. & Toth, Eric \\
\hline Jena, Prasant & Touboul, Cyril \\
\hline Jendelova, Pavla & Tous, Nuria \\
\hline Jeng, Jiiang-Huei & Tovoli, Francesco \\
\hline Jenkins, Jill A. & Toy, Randall \\
\hline Jenkins, Johnie Norton & Toyoda, Atsushi \\
\hline Jensen, Benjamin & Toyoda, Yu \\
\hline Jensen, Boye L. & Trabace, Luigia \\
\hline
\end{tabular}




\begin{tabular}{|c|c|}
\hline Jensen, Eric & Tramontano, Donatella \\
\hline Jensen, Lars Henrik & Tran, Kim \\
\hline Jensen, Randy L. & Tran, Pamela \\
\hline Jeong, Daewon & Tran, Thach D. \\
\hline Jeong, Keun-Yeong & Tranbarger, Timothy \\
\hline Jeong, Kyoung Yong & Trant, John \\
\hline Jeong, Seon-Yong & Trapani, Joseph A. \\
\hline Jeschke, Marc & Trautinger, Franz \\
\hline Jeschke, Udo & Travlos, Ilias \\
\hline Jesenak, Milos & Traw, Milton Brian \\
\hline Jesionowski, Teofil & Trdan, Stanislav \\
\hline Jha, Amit & Treesukosol, Yada \\
\hline Ji, Peng & Treglia, Giorgio \\
\hline Jiang, Shu-Ye & Trejo, Joann \\
\hline Jiang, Sizun & Tremblay, Marie-Ève \\
\hline Jimenez-Rondan, Felix & Trenti, Annalisa \\
\hline Jin, Dong-Hoon & Trentini, Alessandro \\
\hline Jin, Fan & Tresguerres, Jesús \\
\hline Jin, Jiefu & Trevisan, Andrea \\
\hline Jin, Ran & Trevisan, Giusto \\
\hline Jin, Xin & Trevisi, Lucia \\
\hline Jinesh, Goodwin G. & Triaca, Viviana \\
\hline Jinnouchi, Hideaki & Trichet, Valérie \\
\hline Jo, Young Suk & Tricoire, Ludovic \\
\hline Job, Dominique & Trif, Monica \\
\hline Jobling, Phil & Trilling, Mirko \\
\hline Joers, Valerie & Tringali, Corrado \\
\hline Johansson, Pegah & Trivedi, Chinmay M. \\
\hline Johansson, Staffan & Trivedi, Darshan \\
\hline John, Rohan & Trivedi, Evan R. \\
\hline John, Varghese & Troczka, Bartlomiej J. \\
\hline Johnson, Thomas V. & Troiano, Giuseppe \\
\hline Johnson, Xenie & Troise, Antonio Dario \\
\hline Johnston, Michael & Trojanowicz, Bogusz \\
\hline Johnstone, Elizabeth & Trombetta, Domenico \\
\hline Joles, Jaap & Troppmair, Jakob \\
\hline Jolly, Pawan & Trovato, Guglielmo \\
\hline Jones, T. Bucky & Trovik, Jone \\
\hline Jonkers, Ilse & Trujillo, Marco \\
\hline Jonnalagadda, Subash C. & Trujillo-Rodríguez, María José \\
\hline Jonsson, Lisbeth M.V. & Truman, Andrew \\
\hline Jonušienè, Violeta & Trummer, Olivia \\
\hline Joo, Kyeung Min & Truong Phuoc, Nghia \\
\hline
\end{tabular}




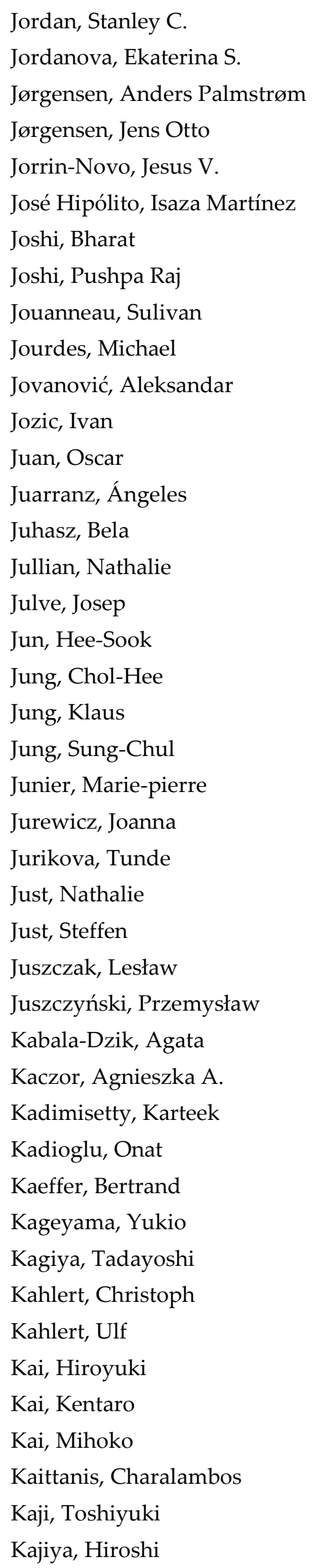

Tsai, James

Tsai, Kun-Chih

Tsai, Kuo-Wang

Tsai, Shih-Jen

Tsang, Suk-Ying

Tschierlei, Stefanie

Tse, William K. F.

Tseng, Ching-Li

Tsirigotis, Panagiotis

Tsitsilonis, Ourania E.

Tsoulfas, George

Tsubaki, Kazunori

Tsuchiya, Yuichi

Tsuji, Masahiro

Tsuji, Shoji

Tsunematsu, Tomomi

Tsurkan, Mikhail V.

$\mathrm{Tu}$, Min

$\mathrm{Tu}$, Thomas

Tuan, Tai-Lan

Tuda, Midori

Tullio, Vivian

Tunaru, Sorin

Tuomisto, Jouko

Tuorto, Francesca

Turck, Natacha

Turco, Jenifer

Turesky, Robert

Turiel, Maurizio

Turnbull, D.

Turpin-Nolan, Sarah

Turrini, Eleonora

Turroni, Silvia

Tuthill, Tobias J.

Tuttolomondo, Antonino

Tvrdik, Petr

Tyagi, Rahul

Tyakht, Alexander V.

Tyan, Yu-Chang

Tyerman, Stephen D.

Tzakos, Andreas G.

Tzfadia, Oren

Uchida, Shizuka 
Kakinuma, Yoshihiko

Kako, Tetsuya

Kalani, Anuradha

Kale, Abhijit

Kalinin, Vladimir I.

Kallunki, Tuula

Kalogiannis, Konstantinos G.

Kalogirou, Charis

Kamachi, Kazunari

Kamada, Yoshihiro

Kamagata, Kiyoto

Kamal, Abu Hena M.

Kamath, Sandip

Kamato, Danielle

Kamiloglu, Senem

Kamisuki, Shinji

Kamitani, Shigeki

Kamiya, Mitsunobu

Kamnev, Alexander

Kamolz, Lars

Kamphuis, Lars G.

Kanakkanthara, Arun

Kanamori, Takao

Kanaoka, Masahiro

Kanasaki, Keizo

Kandimalla, Raju

Kanemori, Masaaki

Kang, Chang Moo

Kang, Chen

Kang, Hee

Kang, Hunseung

Kang, Lei

Kang, Sinyoung

Kang, Tae-Hong

Kang, Wei

Kanie, Osamu

Kanik, Mehmet

Kaňková, Kateřina

Kannan, Ram

Kano, Mitsuyoshi

Kant, Merijn

Kanvil, Sadia

Kanzaki, Hiroyuki
Uchida, Takeshi

Uchihashi, Takayuki

Uchio, Yuji

Ueda, Hiroshi

Ueha, Satoshi

Ueno, Shu-ichi

Ueno, Takafumi

Ufnal, Marcin

Uhal, Bruce D.

Uhl, Eberhard

Ulisse, Salvatore

Ulivieri, Cristina

Ulloa, Rita

Ulrich, M.M.W.

Umberger, Reba A.

Umehara, Mikihisa

Ungefroren, Hendrik

Unno, Keiko

Upadhyaya, Indu

Urban, Jill P.G.

Urbanelli, Lorena

Urbano, A. M.

Usui, Michihiko

Uusitupa, Matti

Uysal, Utku

Václav, Brázda

Václavíková, Radka

Vago, Riccardo

Vähä-Koskela, Markus

Vaidya, Bhuvaneshwar

Vaidyanathan, Ganesan

Valdes, Ana

Vale, Nuno

Valensin, Daniela

Valentina, Vaira

Valentino, Mario

Valentová, Kateřina

Valentovic, Monica

Valenzuela, Rodrigo

Valério, Elisabete

Valero, Daniel

Vali, Payam

Valles, Soraya L. 
Kao, Erl-Shyh

Kapitsinou, Pinelopi

Kaplan, Craig

Kappachery, Sajeesh

Kappeler, Laurent

Kappen, Claudia

Kapus, András

Karakasidis, T. E.

Karakochuk, Crystal

Karaman, Sinem

Kararigas, Georgios

Karlic, Heidrun

Kårlund, Anna

Karmakar, Mausita

Karni, Rotem

Karpas, Abraham

Karran, Peter

Karras, Spiros

Karreth, Florian A.

Karvinen, Sira

Kaschina, Elena

Kashfi, Khosrow

Kashiwagi, Shinichiro

Kashiwakura, Ikuo

Kashman, Yoel

Kashofer, Karl

Kasiotis, Konstantinos M.

Kasoju, Naresh

Kassiri, Zamaneh

Kasten-Jolly, Jane

Kataoka, Naoyuki

Katarina, Stroffekova

Katase, Naoki

Kathuria, Himanshu

Kato, Takamitsu A.

Kato, Yasumasa

Katoh, Masaru

Katsantonis, Dimitrios

Katsarava, Ramaz

Katsila, Theodora

Katsoris, Panagiotis

Kaufman, Brett

Kaufmann, Dorothea
Valverde, Federico

Van Aken, Olivier

Van Ballegooijen, Adriana J

Van Bergen En Henegouwen, Paul M.P.

Van Beuningen, Henk M.

Van Bever, Yolande

Van Coppenolle, Fabien

Van Cruchten, Steven J.

Van Cutsem, Pierre

Van De Ven, Rieneke

Van Den Bergh, Hubert

Van Den Noort, M. W.M.L.

Van Der Kuyl, Antoinette

Van Der Laarse, Willem J.

Van Der Mei, Ingrid A.f.

Van Der Spoel, Aarnoud C.

Van Der Straaten, Tahar

Van Der Veer, Eric P.

Van Der Weijden, Renata

Van Eps, Andrew Van Eps

Van Eys, Guillaume J.

Van Geijlswijk, Ingeborg M.

Van Hall, Thorblad

Van Leeuwen, Hans

Van Lent, Peter LEM

Van Loo, Hanna M.

Van Noorden, Cornelis

Van Rijn, Richard M.

Van Roosbroeck, Katrien

Van Scherpenzeel, Monique

Van Spaendonck-Zwarts, K. Y.

Van Vliet, Sandra

Van Waardenburg, R. C. A. M.

Vancurova, Ivana

Vandenwijngaert, Sara

Vanderheyden, Patrick

Vandier, Christophe

Vandooren, Jennifer

Vanduffel, Wim

Vangelista, Luca

Vankemmelbeke, Mireille

Vankova, Radomira

Vanneaux, Valérie 
Kaunas, Roland R.

Kauppinen, Anu

Kaur, Jasmine

Kawabata, Takeshi

Kawada, Manabu

Kawahata, Kimito

Kawai, Vivian K.

Kawai, Yoshichika

Kawanami, Daiji

Kawano, Kouichiro

Kawano, Tomonori

Kawasaki, Hideya

Kawasaki, Ichiro

Kawasumi, Masaoki

Kawiak, Anna

Kayama, Masazumi

Kayisli, Umit

Kazeto, Yukinori

Kazlauskas, Andrius

Kazunori, Kadota

Kearsey, Stephen

Kedrov, Alexej

Keku, Temitope

Keller, Simona

Kelley, Darshan

Kelley, Eric

Kelly, Clive Anthony

Kenna, Dervla T.D.

Kenneth Matthew, Scaglione

Kerwin, Rachel E.

Keshteli, A. H.

Kessler, Sonja

Kevadiya, Bhaveah

Kevadiya, Bhavesh

Keyaerts, Marleen

Khadka, Manoj

Khalid, Syma

Khalil, Abdelouahed

Khaliulin, Igor

Khallouki, Farid

Khan, Mohsin

Khan, Muhammad

Khan, Mushfiquddin
Vanneste, Steffen

Vapaatalo, Heikki

Vaquero, Javier

Varando, Gherardo

Varela-Nieto, Isabel

Vasaikar, Suhas

Vashist, Yogesh Kumar

Vasicek, Ondrej

Vasin, Mikhail V.

Vassalle, Cristina

Vassilaki, Niki

Vassilopoulos, George

Vaughan, Roger A.

Vaughn, Byron

Vaughn, Mathew

Vaya, Jacob

Vaz, Deisi Altmajer

Vaz, Josiana

Vazquez-Manrique, Rafael

Vecchione, Carmine

Vecoli, Cecilia

Veenstra, Richard D.

Veeramani, Suresh

Veiga-Lopez, Almudena

Veilleux, Alain

Veis, Arthur

Vejux, Anne

Velasco Ortega, Eugenio

Velasco, Guillermo

Veldkamp, Christopher

Veleeparambil, Manoj

Velena, A.

Velivelli, Siva

Vellante, Federica

Velliyagounder, Kabilan

Vemula, Harika

Venditti, Alessandro

Vento, Renza

Venuti, Aldo

Verdaguer, Dolors

Verdoucq, Lionel

Vergadi, Eleni

Vergara, Daniele 
Khandaker, Morshed

Khare, Vineeta

Khasawneh, Fadi

Khatir, Dinah S.

Khatodia, Surender

Khatri, Kshitij

Khatri, Mahesh

Khetani, Salman

Khoo, Bee Luan

Khoobchandani, Menka

Khoshmanesh, Khashayar

Khotimchenko, Maxim Y.

Khurshid, Zohaib

Kibe, Toshiro

Kibriya, Muhammed

Kichkailo, Anna

Kido, Mizuho A.

Kiely, Patrick

Kilari, Sreenivasulu

Killilea, David

Kim, Bong-Sung

Kim, Dong Joon

Kim, E. Edmund

Kim, Haeng-Hoon

Kim, Hangun

Kim, Hong-Hee

Kim, Hoon

Kim, Hun Sik

Kim, Hyun Uk

Kim, Hyung-Goo

Kim, Hyung-Joon

Kim, InKyeom

Kim, Jaehan

Kim, Jaehwan

Kim, Jeong-Rae

Kim, Ji Yeon

Kim, Jin Moon

Kim, Jin-Kyung

Kim, Jinu

Kim, Jin-Wook

Kim, Joungmok

Kim, Jung-Ae

Kim, JwaJin
Verges, Marcel

Verhage, Leonie

Verhoeven, Adrie

Vernetti, Lawrence A.

Verron, Elise

Verstraete, Alain

Vervaet, Benjamin

Veskoukis, Aristidis S.

Vesper, Stephen

Vetere, Amedeo

Vetter, Stefan W.

Vetvicka, Vaclav

Vetvickova, Jana

Viapiana, Agnieszka

Vicente, Claudia

Vicente, Joana G.

Vicente, Laura

Victoni, Tatiana

Victor, Bruno

Videira, Romeu António

Vieira Ribeiro, João Rui

Vieira, Margarida C.

Viejo-Borbolla, Abel

Viel, Sébastien

Viennois, Emilie

Viggiano, Andrea

Viggiano, Davide

Vigueira, Cynthia

Vijayavenkataraman, S.

Vilageliu, LLuisa

Vilela, Alice

Villa, Roberto Federico

Villa-Bellosta, Ricardo

Villegas-Sepúlveda, Nicolas

Vincent, Jean-louis

Vinciguerra, Manlio

Vinnedge, Lisa Privette

Vinogradov, Evguenii

Viola, Ivana

Virtue, Sam

Visani, Michela

Visentin, Michele

Visioli, Francesco 
Kim, Ki Hyun

Kim, Kil-Soo

Kim, Kyoung Soo

Kim, Kyounghyun

Kim, Kyung-su

Kim, Nam-Jung

Kim, Sang-We

Kim, Sunggil

Kim, Sung-Hoon

Kim, Sun-Ju

Kim, Tae Il

Kim, Tae-Aug

Kim, Woo-Yang

Kim, Young Jo

Kim, Yun-bae

Kimbrel, Erin

Kimler, Bruce

Kimura, Mayumi

Kimura, Nobuyuki

Kimura, Tomoki

Kinaciyan, Tamar

King, Chih-Yen

King, Irena B.

Kinghorn, Kerri J.

Kingsford, Carl

Kinsey, William $\mathrm{H}$.

Kirby, Lynn Guertin

Kirker, Grant

Kirkham, M. B.

Kirsch, Thorsten

Kiselev, K. V.

Kishigami, Satoshi

Kishimoto, Yoshimi

Kita, Hirohito

Kitaoka, Satoshi

Kitatani, Kazuyuki

Kitchen, Philip

Kittaka, Atsushi

Kiyan, Yulia

Klampfer, Lidija

Klampfl, Stefanie

Klaus, Susanne

Klein, David C.
Visser, Jacob

Visser, Lydia

Vita, Roberto

Vitale, Giovanni

Vítámvás, Pavel

Vitetta, Luis

Vittorioso, Paola

Vivash, Lucy

Vladimirov, Vladimir

Vlahou, Antonia

Vliagoftis, Harissios

Vo, Minh D.

Vodicka, Pavel

Voelkers, Mirko

Vogel Ciernia, Annie

Vogelaar, Christina Francisca

Vogt, Martin

Voitsekhovskaja, Olga V.

Volk, David

Volk, Susan W.

Volz, David C.

Von Knethen, Andreas

Von Schacky, Clemens

Von Wright, Atte

Vona, Barbara

Vonk, Lucienne A.

Vorsters, Alex

Voss, Matthias

Vossen, Jack H.

Vostinaru, Oliviu

Vrana, Nihal Engin

Vriend, Jerry

Vrsaljko, Domagoj

Vrtala, Susanne

Vu Bac, Nam

Vucenik, Ivana

Vuckovic, Dajana

Vugrek, Oliver

Vulliamy, Thomas

Vuong, Thu V.

Vuorimaa-Laukkanen, Elina

Wada, Jun

Wade, Charles E. 
Klein, Stefan

Klempt, Martin

Kleppe, Lene

Klimaschewski, Lars

Kline, Kimberly

Kłoda, Karolina

Klokov, Dmitry Y.

Klontzas, Michael

Klose, Johannes

Klosterman, Steven J.

Klug, Jörg

Klutsch, Jennifer

Kluza, Jérome

Kmetič, Ivana

Kmiec, Eric

Kmiec, Eric B.

Kmiecik, Justyna

Knaggs, Roger

Knecht, Hans

Knetsch, Menno L. W.

Knetsch, Menno L.W.

Knežević, Sanda Vladimir

Knight, Jason S.

Kniss, Douglas A.

Knölker, Hans-Joachim

Knott, K. Emily

Knutsen, Svein

Kobayashi, Hideki

Kobayashi, Satoru

Kobayashi, Yayoi

Kobayashi, Yoshiro

Kobayashi, Yutaro

Kobeissy, Firas H.

Köbel, Martin

Kocarnik, Jonathan M.

Koch, Alexander

Koch, Marco

Koduru, Srinivas V

KOENEN, Rory R.

Koga, Jun-ichiro

Kohanbash, Gary

Köhl, Gudrun

Kohl, Matthias
Wadehra, Madhuri

Wadsak, Wolfgang

Wagner, Anika

Wagner, David

Wagner, Gerd K.

Wahlestedt, Claes

Wahli, Walter

Wahyudi, Hendra

Waldmeier, Felix

Walentek, Peter

Waleron, Krzysztof

Walker, Chandler L.

Walker, Douglas G.

Walker, Jessica

Wallace, David

Wallace, Nicholas

Walne, Amanda J.

Walsh, Laurence

Walters, Dianne

Walts, Ann E.

Wan, Lei

Wan, Shibiao

Wan, Xuehua

Wanders, Desiree

Wang, Chao-Min

Wang, Chi Chiu

Wang, Ching-Chiung

Wang, Chrong-Reen

Wang, ChuanFeng

Wang, Dandan

Wang, David

Wang, Dong

Wang, Feng-Sheng

Wang, Guozheng

Wang, Hai

Wang, Huaimin

Wang, J.

Wang, James $Q$.

Wang, Jennifer

Wang, Jian-Wen

Wang, Jun

Wang, Junjie

Wang, Liang 
Kohri, Michinari

Koivisto, Ari

Kojima, Masami

Kojima, Naoya

Kojima, Shihoko

Kojima-Yuasa, Akiko

Kok, Dieuwertje

Kok, Victor C.

Kok, Wouter

Kokabu, Shoichiro

Kolbe, L.

Koller, Martin

Koloniuk, Igor

Kolpen, Mette

Komasa, Satoshi

Komatsu, David

Komine, Mayumi

Komoike, Yuta

Komorowski, Jan

Kondo, Eisaku

Kondo, Hidehiro

Koneru, Bhuvaneswari

Kong, Weili

Konig, Heiko

Koning, Dirk De

Konno, Katsuhiro

Konopka-Postupolska, D.

Konstantinov, Spiro M.

Kontny, Udo

Kontogiannatos, Dimitrios

Kooiman, Klazina

Kootala, Sujit

Kopecki, Zlatko

Kopel, Pavel

Koppe, Janna G.

Koppel, Barbara S.

Korasick, David A

Koren III, John

Kornek, Miroslaw

Kornprat, Peter

Korrodi-Gregório, Luís

Korsching, Eberhard

Kosaka, Nobuyoshi
Wang, Meinan

Wang, Peng-Hui

Wang, Piwen

Wang, Raymond Y.

Wang, Richard

Wang, Robert Y. L.

Wang, San-Lang

Wang, Sheng

Wang, Sheng-Fan

Wang, Shyi-wu

Wang, Tian-Li

Wang, Tong-Hong

Wang, Tuo

Wang, Tzu-hao

Wang, Wei-Lien

Wang, Wei-Lung

Wang, Wentian

Wang, Xiaofei

Wang, Xiaohong

Wang, Xiaowan

Wang, Xin

Wang, Xinkun

Wang, Xinwen

Wang, Xiping

Wang, Xuejun

Wang, Yanchang

Wang, Yumeng

Wang, Yunmei

Wang, Yunxiao

Wang, Yuqi

Wang, Zhanxiang

Wang, Zhe

Wang, Zhijie

Wanibuchi, Hideki

Ward, Christopher

Ward, Peter A

Warnecke, Athanasia

Warren, Sean

Warzecha, Zygmunt

Wasternack, Claus

Watanabe, Atsushi

Watanabe, Eiichi

Watanabe, Masatoshi 
Koskela, Ali

Koskinen Holm, Cecilia

Koslicki, David

Kosmala, Arkadiusz

Kosova, Klara

Kostakis, Ioannis

Kostin, Sawa

Kotake, Shigeru

Kotloski, Robert

Kotsikorou, Evangelia

Kotta-Loizou, Ioly

Kotula-Balak, Malgorzata

Koturbash, Igor

Kounis, Nicholas G

Koupenova, Milka

Kourist, Robert

Kousoulas, Konstantin G.

Koutelidakis, Antonios

Kovac, Stjepana

Kovacic, Hervé

Kovács, Ákos T.

Kovalsky, Ilona

Kovalskyy, Dmytro

Kovvasu, Surya

Kowalska, Malgorzata

Kowarik, Markus C.

Kox, Matthijs

Koyama, Shin

Koyama, Yu

Kozaki, Akiko

Koziak, Katarzyna

Kozliak, Evguenii I.

Kozlowski, Michael R.

Kramer, Eric

Kramer, Phillip

Krammer, Florian

Krasnodembskaya, Anna D.

Kratimenos, Panagiotis

Kraus, Virginia

Krause, Rolfdieter

Krauss, Daniel J.

Kregel, Steven

Křen, Vladimír
Waterman, Carrie

Waters, Paula J.

Watkins, Adam J.

Watzka, Matthias

Waugh, Mark G.

Wawer, Iwona

Webb, Kimberly M.

Weber, Michael J.

Weeden, Norman F.

Wege, Stefanie

Wegner, Marthe-Susanna

Wei, Changyong

Wei, Gan

Wei, Qiu

Wei, Ren

Wei, Sai-nan

Wei, Shau-Ming

Wei, Yufeng

Weichert, Jamey

Weiergräber, Marco

Weigand, Annika

Weigand, Julia E.

Weihua, Zhang

Weiser, Douglas C.

Weivoda, Megan

Welch, Babu Guai

Welch, Carrie

Wellberg, Elizabeth A

Weller, Michael

Wellner, Ulrich

Welsch, Ralf

Welsh, Michael

Wen, C.M.

Wen, Lu

Wen, XIAOMIN

Wende, Wolfgang

Wendell, Stacy Gelhaus

Weng, Chingfeng

Weng, Mao-Lun

Weng, Xiaoyu

Werstuck, Geoff H.

Wertz, Philip W.

West, James D. 
Krenek, Pavel

Krepler, Clemens

Krisch, Judit

Krischik, Vera A

Krishna, Smriti

Krishnan, Laxminarayanan

Kristensen, Mie

Kristian, Tibor

Krogan, Naden T.

Krohn, Sandra

Krol, Ewelina

Kroncke, Brett $\mathrm{M}$.

Kropp, Martina

Kropski, Jonathan A.

Krützfeldt, Jan

Kruyt, Frank A. E.

$\mathrm{Ku}$, Kang Mo

$\mathrm{Ku}$, Seockmo

$\mathrm{Ku}$, Seung-Yup

Kuan, Yu-Hsiang

Kuang, Huihui

Kubatka, Peter

Kubik, Lukasz

Kubiński, Konrad

Kubista, Helmut

Kubo, Takanori

Kubo, Tomohiko

Kubo, Toshio

Kucukkal, Tugba

Kuhlmann, Stella

Kui, Balázs

Kukula-Koch, Wirginia

Kumar, Binod

Kumar, Dhirendra

Kumar, Gaurav

Kumar, Gokhlesh

Kumar, Manish

Kumar, Manu

Kumar, Mukesh

Kumar, Rajive

Kumar, Sathish

Kumar, Sushil

Kumar-Singh, Samir
West, Tim

Westenfelder, Christof

Westin, Gunnar

Whelan, Rebecca J.

Whirledge, Shannon

White, Peter

White, Stormi Pulver

Whiteside, Teresa L.

Whyard, Steve

Wickenden, Alan D.

Wiczkowski, Wieslaw

Widdison, Wayne C.

Widgerow, Alan

Wie, Myung-Bok

Wiechec, Emilia

Wiechmann, Allan

Wiedenmann, Joerg

Wiedman, Gregory

Wiemer, Erik A.C.

Wierenga, Corette J.

Wierzchowski, Marcin

Wieser, Rotraud

Wiesner-Reinhold, Melanie

Wietrzyk, Joanna

Wigle, Jeffrey T.

Wild, Edward

Wildemann, Britt

Wilhelm, Stefan

Wilkinson, Mark D.

Wilkinson, Robert

Willcox, Mark

Williams, Adina

Williams, Andrew R.

Williams, Cecilia

Williams, David

Williams, John

Williams, Noelle S.

Williams, Thomas L.

Williams, Trevor

Williamson, Sean R.

Wilmink, Johanna W.

Wilson, Douglas

Win, Khin Yin 
Kumazawa, Yoshinori

Kumi-Diaka, James

Kumita, Janet

Kummer, Wolfgang

Kundu, Aishwarya

Kunej, Tanja

Kunjachan, Sijumon

Kunkalla, Kranthi

Kunz, Wolfram

Kunze, Gotthard

Künzler, Markus

Kuo, Cheng-Chin

Kuo, Ping-Chung

Kuo, Po-Lin

Kuo, Tzong-Fu

Kurakula, Kondababu

Kurdowska, Anna K.

Kurenda, Andrzej

Kurokawa, Mineo

Kurschus, Florian

Kurz, Tino

Kuter, Katarzyna

Kuwabara, Masanari

Kuyucu, Semanur

Kuźnicki, Jacek

Kveberg, Lise

Kwa, Faith A.A.

Kwak, Minjung

Kwakowsky, Andrea

Kwan, Hiu-yee

Kwan, James

Kweon, Oh-Kyeong

Kwiecien, Jacek M.

Kwon, Hyokjoon

Kwon, Tae-Hwan

Kwon, Tae-yub

Kwong, Joseph

Kyle, Stuart

La Rocca, Renato V.

La Russa, Daniele

Laboisse, Christian L.

Labruna, Giuseppe

Lachman, Jaromir
Winkler, Johannes

Win-Shwe, Tin-Tin

Wińska, Katarzyna

Winter, Harland

Winter, Stephane

Winterbourn, Christine

Winters, Bryony

Wirbisky, Sara

Wise, John

Wise, Petra

Wis"niowska, Barbara

Witkowski, Marco

Witorsch, Raphael Jay

Witowski, Jan Sylwester

Witten, Paul Eckhard

Witt-Enderby, Paula

Wittstock, Ute

Wittwer, Carl

Wnuk, Maciej

Wobbe, Lutz

Wohleb, Eric S.

Wohlert, Jakob

Wojcieszyńska, Danuta

Wójkowska, D. W.

Wojtaszek, Przemyslaw

Wojtkiewicz, Joanna

Wölfle, Ute

Wolley, Martin

Wong, Albert H.C.

Wong, Boon-Seng

Wong, Ching-On

Wong, Chung F.

Wong, Connie

Wong, Pamela T.

Wong, Ronald J.

Wong, Shun

Wong, Vincent Kam Wai

Woo, So-Youn

Woodman, Tim

Woods, Nicholas

Woolbright, Ben

Worch, Remigiusz

Workman, Victoria 
Lacis, Gunars

Ladavière, Catherine

Lærke, Helle

Lafontaine, Denis

Laforenza, Umberto

Lagace, Thomas A.

Lagravère, Manuel $\mathrm{O}$.

Lagrimini, Mark

Lahiri, Amit

Lahiri, Amitabha

Lahooti, Hooshang

Lai, Julian C. L.

Lai, Wen-Fu Thomas

Lai, Yandong

Laird-Offringa, Ite A.

Lakhkar, Anand

Lakin-Thomas, Patricia L.

Lalonde, Francois

Lalowski, Maciej

Lam, Hon-Ming

Lam, Wai-Ching

LAM, Yulin

Lamarche-Vane, Nathalie

Lamas, José Ramón

Lamb, Laura

Lamina, Claudia

LaMontagne, Michael

Lan, Lan

Landete, José Maria

Landreh, Michael

Landreville, Solange

Landry, Samuel J.

Laner-Plamberger, Sandra

Lange, Dirk

Lange, Sigrun

Langkjær, Niels

Lanz, Tobias V.

LaPres, John J.

Lapucci, Cristina

Lardone, Patricia J.

Larena, Inmaculada

Larijani, Mani

LaRoque, Jan
Wozniak-Knopp, Gordana

Wozniakowski, Grzegorz

Wren, Brendan W.

Wright, Catherine

Wright, Colin

Wrzaczek, Michael

$\mathrm{Wu}$, Chengbiao

Wu, Chi-Rei

$\mathrm{Wu}$, Christina

Wu, Hannah

$\mathrm{Wu}$, Jiahui

Wu, Kevin C. W.

$\mathrm{Wu}$, Tzong-Yuan

$\mathrm{Wu}$, Wen-Sheng

$\mathrm{Wu}$, Yunqi

Wullaert, Andy

Wydau, Sandra

Wysocki, Annette

Xavier, Cristina Pinto Ribeiro

$\mathrm{Xi}$, Lin

$\mathrm{X}$, Weixian

Xia, Tian

Xian, Wa

Xiang, Yu

Xiao, Junhua

Xiao, Li

Xiao, Yangyan

Xiao, Yuguo

Xiaoli, Alus

Xie, Hong

Xie, Jinghang

Xie, Qi

Xie, Zhong-Ru

Xin, Dongyue

Xin, Junna

Xin, Ran

Xing, Jinchuan

Xing, Zhuo

Xiong, Ye

Xiong, Zhaohui

$\mathrm{Xu}$, Deyang

$\mathrm{Xu}$, Haineng

$X u$, Lei 


\begin{tabular}{|c|c|}
\hline Larraga, Vicente & $\mathrm{Xu}$, Pengfei \\
\hline Larsen, Anders Christian & Xu, Qinqin \\
\hline Lasky-Su, Jessica Ann & $\mathrm{Xu}$, Wang \\
\hline Lassmann, Hans & $\mathrm{Xu}$, Yanyi \\
\hline Lassot, Iréna & $X u, Y i$ \\
\hline Latella, Giovanni & $\mathrm{Xu}$, Yongjie \\
\hline Latorre, Juan David & Xuan, Tran Dang \\
\hline Lattanzio, Rossano & Yaegashi, Hajime \\
\hline Lau, Wei Ling & Yager, Eric \\
\hline Laubach, Victor E. & Yagüe, Ernesto \\
\hline Lau-Cam, Cesar A. & Yakovlev, Igor A. \\
\hline Launay, Jean Marie & Yamada, Sohsuke \\
\hline Lauretani, Fulvio & Yamada, Yasumasa \\
\hline Lauro, Clotilde & Yamaguchi, Masaya \\
\hline Lauschke, Volker & Yamaguchi, Seiji \\
\hline Lavandera, Iván & Yamaguchi, Yube \\
\hline Lavarino, Cinzia & Yamakoshi, Kimi \\
\hline Lavelli, Vera & Yamamoto, Hideyuki \\
\hline Laviano, Alessandro & Yamamoto, Yusuke \\
\hline Lavoie, Jean-claude & Yamamotova, Anna \\
\hline Lavrik, Olga & Yamamura, Soichiro \\
\hline Lawlor, Kate & Yamanishi, Kiyofumi \\
\hline Layh-Schmitt, Gerlinde & Yamasu, Kyo \\
\hline Lazado, Carlo C. & Yamauchi, Yohei \\
\hline Lazarević, Vladimir & Yan, Bowen \\
\hline Le Bideau, Franck & Yan, Dayun \\
\hline Le Bihanic, Florane & Yan, Dongqing \\
\hline Le Hir, Hervé & Yan, Qing \\
\hline Le Hir, Rozenn & Yanase, Emiko \\
\hline Le Ouay, Benjamin & Yang, Chin-Ying \\
\hline Le, Minh & Yang, Guang \\
\hline Leanza, Luigi & Yang, Haining \\
\hline Lebar, Matthew & Yang, Jae Wook \\
\hline Lebaron, Richard & Yang, Jen-Chang \\
\hline Lebaron, Simon & Yang, Jenq-Lin \\
\hline Lebeck, Janne & Yang, Jun \\
\hline Lebedev, Albert T. & Yang, Kuang-Yao \\
\hline Leborgne-Castel, Nathalie & Yang, Rong-Sen \\
\hline Lebrun, Bruno & Yang, Shengyong \\
\hline Lechel, Andre & Yang, Sherry X. \\
\hline Lechpammer, Mirna & Yang, Tao \\
\hline Leclercq, Guy & Yang, Tianxin \\
\hline Ledo, Ana & Yang, Tuo \\
\hline
\end{tabular}


Lee, Byung C.

Lee, ChangWoo

Lee, Che-Hsin

Lee, Chen-Yu

Lee, Choon-Hwan

Lee, Chul-Ho

Lee, Dong Hun

Lee, Hak Jong

Lee, Heung-Man

Lee, Hsinyu

Lee, Huei-Jane

Lee, Je Min

Lee, J-H

Lee, Jin-Kyun

Lee, Jong Hun

Lee, Joo Hyoung

Lee, Ju Hee

Lee, Jun Sik

Lee, Jungil

Lee, Jungwoo

Lee, Kin Wah Terence

Lee, Kyung-Yil

Lee, Kyuwan

Lee, Maw-Rong

Lee, Min-Geol

Lee, Myung Koo

Lee, Sang Kil

Lee, Sang-Han

Lee, Sanghyeob

Lee, Sangkwan

Lee, Soohong

Lee, Su-Jae

Lee, Sukmook

Lee, Tzong-Shyuan

Lee, Wing-Kee

Lee, Yi-Jang

Lee, Yoon Kwang

Lees, Watson J.

Lefebvre, Hervé

Leffler, Jonatan

Lehmann, Christian

Lei, Lei

Leibundgut-Landmann, S.
Yang, Wei-hsiung

Yang, Xinmai

Yang, Ye

Yang, Yongkang

Yang, Yunze

Yano, Shozo

Yano, Tohru

Yao, Jian

Yao, Jianbo

Yao, Jiayi

Yao, Yanhua

Yar, Muhammad

Yasuda, Kazuki

Yasuda, Takako

Yasuike, Motoshige

Yates, Nathanael J.

Ye, Haobin

Ye, Zhou

Yeckel, Catherine Weikart

Yeh, Jwu-Lai

Yeh, Sung-Ling

Yeh, Yin-Ting

Yektaei-Karin, Elham

Yeligar, Samantha

Yellen, Gary

Yen, Meng-Chi

Yeo, Syn

Yeung, Sai-Ching Jim

Yi, Haowei

Yih, Ling-huei

Yilmazer, Açelya

Yin, Jingwen

Yin, Kingsley

Yin, Shi

Yli-Mattila, Tapani

Yochum, Gregory S.

Yohei, Shirakami

Yokohira, Masanao

Yokoi, Hideki

Yokoi, Tsuyoshi

Yokota, Shinso

Yoneda, Toshiyuki

Yonekura, Shinichi 
Leidenheimer, Nancy

Leiherer, Andreas

Leitão, J. H. G.

Leitz, Jeremy

Lekli, Istvan

Lemu, Hirpa G.

Lenaerts, Kaatje

Lenardon, Megan D.

Lendrem, Dennis

Leng, Roger

Lennox, Kim

Lensink, Marc F.

Leon, Carlos

Leon, Josefa

Leonard, Stephen

Leone, Alessandro

Leoni, Alberto

Leon-Reyes, Antonio

Leporatti, Stefano

Lerm, Maria

Leroux, Caroline

Leroy, Baptiste

Leroy, Jérôme

Lescrinier, Eveline

Leso, Veruscka

Letizia, Trovato

Leung, Chung-Hang

Leung, Ricky Yuet-Kin

Leustek, Thomas

Levesque, Celine

Levi, Moshe

Levinson, Ralph D.

Levraud, Jean-pierre

Levy, Steven

Lewandoski, Mark

Lewinska, Anna

Li, Aitao

Li, Benyi

Li, Calvin

Li, Chin

Li, Chuan

Li, Dapeng

Li, Deying
Yoneshiro, Takeshi

Yong, Jeongsik

Yoo, Tag Keun

Yool, Andrea

Yoon, Je-Hyun

Yoong, Michael

Yoshida, Hiroshi

Yoshida, Naoko

Yoshida, Yamato

Yoshimoto, Koji

Yoshino, Jun

Yoshitomi, Hiroyuki

You, Seungkwon

You, Sylvaine

You, Xiaona

Young, David

Young, G. Bryan

Young, Jason C.

Young, Marian

Yovich, John

Yu, Aiming

Yu, Cheng-Ping

Yu, Hak Ki

$Y u$, Meifang

Yu, Yan Ping

Yu, Yang

Yu, Yanlin

Yu, Yingjie

$\mathrm{Yu}$, Yong-ming

Yuan, Chunyan

Yuan, Huipin

Yuan, Xue

Yuan, Zhao

Yuan, Zhihong

Yudoh, Kazuo

Yue, Patrick

Yue, Xiaojing

Yue, Xuyi

Yuen, John Wm

Yuh, Chiou-Hwa

Yukl, Erik T.

Yung, Hong-Wa

Yung, Yuval 


\begin{tabular}{|c|c|}
\hline Li, Feng & Yuzawa, Yukio \\
\hline Li, Guohui & Zabad, Rana K. \\
\hline Li, Hua-Bin & Ząbek, Adam \\
\hline Li, Hui & Zabetakis, Ioannis \\
\hline Li, Huinan & Zafar, Muhammad \\
\hline Li, Jinping & Zagidullin, Naufal \\
\hline Li, Kuo-Bin & Zaid, Hilal \\
\hline Li, Lin & Zajonc, Dirk M. \\
\hline Li, Miaomiao & Zakharian, Eleonora \\
\hline Li, Ming & Žáková, Lenka \\
\hline Li, Ningjun & Zakrzewicz, Dariusz \\
\hline Li, Qiang & Zaky, Ahmed \\
\hline Li, Robert & Zalupski, Mark M. \\
\hline Li, Shiweng & Zamberletti, Erica \\
\hline Li, Wen-Wu & Zambrano-Wilson, Zambrano-Wilson \\
\hline Li, Yan & Żamojć, Krzysztof \\
\hline Li, Yang & Zamyatnin, Andrey A. \\
\hline Li, Yangming & Zang, Liqing \\
\hline Li, Yanjie & Zannetti, Antonella \\
\hline Li, Yimeng & Zara, Severino \\
\hline Li, Yujing & Zaric, Svetislav S. \\
\hline Li, Zhe & Zarogoulidis, Paul \\
\hline Liagre, Bertrand & Zarrelli, Armando \\
\hline Liao, Francesca-Fang & Zarros, Apostolos \\
\hline Liao, Jen-Chung & Zarubaev, Vladimir \\
\hline Liao, Jyh-fei & Zaza, Gianluigi \\
\hline Liao, Yichu & Zehbe, Ingeborg \\
\hline Liao, Yi-Jen & Zeilinger, Carsten \\
\hline Liapi, Charis & Zeilinger, Katrin \\
\hline Libik-Konieczny, Marta & Zeleny, Reinhard \\
\hline Licciardello, Grazia & Zeller, Sebastian \\
\hline Lidon, Fernando & Zellner, Johannes \\
\hline Lifschitz, Carlos & Zeman, Daniel Meier \\
\hline Ligon, Lee A. & Zemel, Michael \\
\hline Ligterink, Wilco & Zenclussen, Ana \\
\hline Lim, Jae Hyang & Zendo, Takeshi \\
\hline Lim, Junxian & Zervakis, Georgios I \\
\hline Lim, Kyu & Zeugolis, Dimitios \\
\hline Lim, Lee Wei & Zevin, Alexander S. \\
\hline Lim, Ming & Zhang, Bo \\
\hline Lim, Shiang Y. & Zhang, Caiguo \\
\hline Lim, Soyeon & Zhang, Chi \\
\hline Lim, Sung-Jig & Zhang, Duo \\
\hline
\end{tabular}


Lima, Sofia A. Costa

Lin, Chen

Lin, Chih-Chien

Lin, Chih-Li

Lin, Daniel

Lin, Hai-shu

Lin, Ho

Lin, Jung-Chun

Lin, Kui

Lin, Kwang-Huei

Lin, Li-Huei

Lin, Nancy

Lin, Pei-Hui

Lin, Qianqian

Lin, Shankung Lin

Lin, Shengda

Lin, Ying-Hung

Lindemann, Peter

Linder, Stig

Lindner, Robert

Ling, Hui

Ling, Maurice

Lingappan, Krithika

Linhardt, Robert

Linninger, Andreas A.

Linseman, Daniel

Lionetti, Vincenzo

Liou, Ying-ming

Lips, Katrin S.

Littlefield, Bruce A.

Litwin, Mieczysław

Liu, Baoming

Liu, Botao

Liu, Chao-Lin

Liu, Cheuk Lun

Liu, Chia-Chi

Liu, Chunming

Liu, Delong

Liu, Ji

Liu, Jianfeng

Liu, Jinghua

Liu, Jun

Liu, Jun-Jen
Zhang, Hengyou

Zhang, Lei

Zhang, Mengliang

Zhang, Qiuyang

Zhang, Sui

Zhang, Tao

Zhang, Tuo

Zhang, Wei

Zhang, Weijie

Zhang, Wenheng

Zhang, Wenjun

Zhang, Wenyu

Zhang, Xuan

Zhang, Yaqing

Zhang, Yixiang

Zhang, Yixuan

Zhang, Yumiao

Zhang, Yuning

Zhang, Yunkai

Zhang, Zhiyong

Zhao, Baoyin

Zhao, Linping

Zhao, Meixia

Zhao, Ningning

Zhao, Qian

Zhao, Qingnan

Zhao, Xiaoai

Zhao, Xuebing

Zhbannikov, Ilya Y.

Zheng, Shilong

Zheng, Wenguang

Zheng, Yue

Zhonping, $\mathrm{Xu}$

Zhou, Bangjun

Zhou, Ben

Zhou, Guo-Ping

Zhou, Heshan Sam

Zhou, Jingjiang

Zhou, Linli

Zhou, Qingxiang

Zhou, Wenchao

Zhou, Xue-Rong

Zhu, Guo-Zhang 
Liu, Junli

Liu, Li

Liu, Pei-Yang

Liu, Rengyun

Liu, Shing-Hwa

Liu, Xiangsheng

Liu, Yang

Liu, Yongqing

Liu, Yu

Liu, Zhenfeng

Liu, Zhixia

Liu, Ziqing

LiWang, Patricia

Ljubimov, Alexander V.

Llobet-Navas, David

Llorens, Eugenio

Lloret, Ana

Lo Muzio, Lorenzo

Lo, Woo Kuen

Loboda, Agnieszka

Lobysheva, Irina

Löffler, Markus

Loganzo, Frank

Lognay, Georges

Loh, Zhixuan

Lohning, Anna

Loinard, Céline

Lombó, Felipe

Lonardo, Amedeo

Longo, Dario Livio

Longoni, Gianluca

Lönn, Peter

Lontok, Erik

Loor, Juan J

Looyenga, Brendan D.

Lopes, Federica
Zhu, Jianhua

Zhu, Jinsheng

zhu, Liandong

Zhu, Qianhao

Zhu, Qiuyu Martin

Zhu, Wei

Zhu, Xiangwei

Zhu, Yuwen

Zhukova, Natalia

Zhuo, Jia L.

Žiarovská, Jana

Zichel, Ran

Zięba, Andrzej

Zienius, Dainius

Zilioli, Samuele

Zimmer, Warren E.

Zimmerlin, Ludovic

Ziv, Meira

Zmijewski, Michal

Żołnowska, Beata

Zonaro, Emanuele

Zorc, Branka

Zorzano, Antonio

Zosky, Graeme

Zoupa, Maria

Zucca, Paolo

Zuhl, Micah

Zukiewicz-Sobczak, W. A.

Züllig, Richard

Zuluaga, Paola

Zuo, Li

Zupko, Istvan

Zuppinger, Christian

Zutz, Christoph

Zuvela, Petar

Zygadlo, Julio 